المجلة الدولية للدراسـات التربوية والنفسية

International Journal of Educational \& Psychological Studies (EPS)

Journal Homepage: https://www.refaad.com/views/EPSR/Home.aspx

www.refaad.com

ISSN: 2520-4149 (Online) 2520-4130 (Print)

\title{
فهم معلمي العلوم لأبعاد الثقافة العلمية وممارستهم لها في ضوء بعض المتغيرات في مديرية تربية عجلون
}

\author{
ولاء مصطفى الصهمادي '، عبدالله محمد خطايبة ’ ، عماد توفيق السعدي

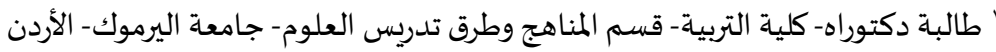 \\ rrrr أستاذ دكتور - كلية التربية- قسم المناهج وطرق تدريس العلوم- جامعة اليرموك- الأردن
}

1 Kulaep_hn@yahoo.com ,2 khataibeh@yu.edu.jo, ,3 Imad.Sadi@yu.edu.jo

DOI: https://doi.org/DOI:10.31559/EPS2020.8.1.3

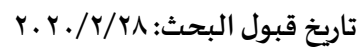

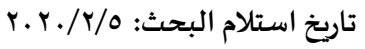

المللخص:

هدفت الدراسة التعرف على مستوى فهم معلمي العلوم لأبعاد الثقافة العلمية وممارستهم لها في ضوء بعض المتغيرات في مديرية تربية عجلون،

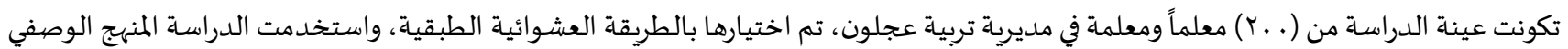

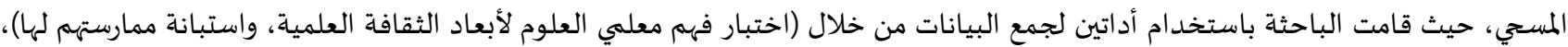

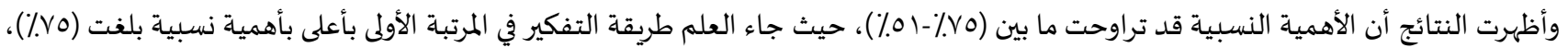

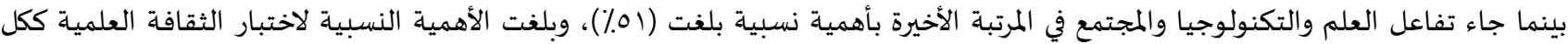

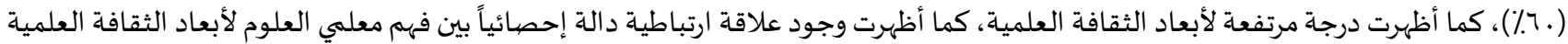
وممارستهم لها، وأظهرت عدم وجود فروق في مستوى فهم معلمي العلوم لأبعاد الثقافة العلمية تبعاً لتخصص، وسنوات الخبرة، والدرجة العلمية،

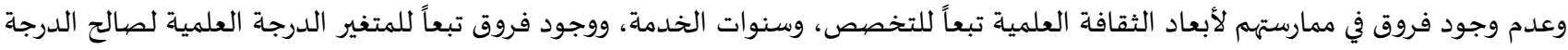
بكالوريوس دبلوم. وأوصت الدراسة تدريب معلمو العلوم أثناء خدمتهم وزيادة الاهتمام المواضيع التي تنمي فهم العلم مثل فلسفة العلوم، وطبيعة

الكلمات المفتاحية: الفهم؛ معلمو العلوم؛ أبعاد الثقافة العلمية. (ब) (1)

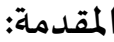

أصبح الاهتمام بالتطور العلمي ضرورة من ضروريات الحياة العصرية، لمواكبة التطورات العلمية والتكنولوجية السريعة، ومواكبة جميع أنظمة الحياة، ونتيجة لهذا التطور فقد أصبح تدربسها في مواد العلوم له أثر بارز وملاحظ. بادون.

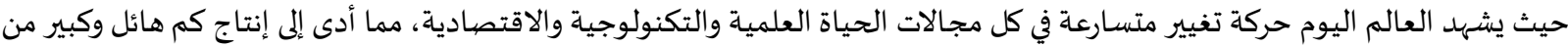

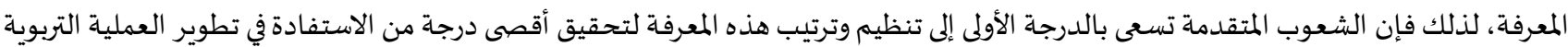

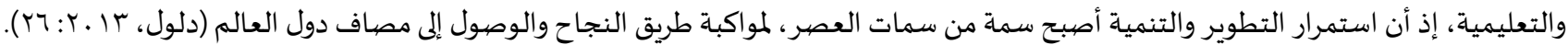

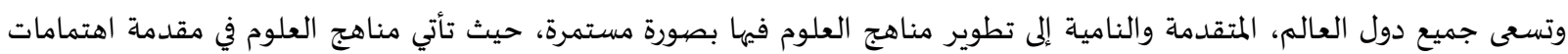
المعنيين، وذلك بوضع سياسات التعليم والتخطيط، لتطويرها، وتحسين مستوى مخرجاتها؛ ويعود ذلك إلى الأهمية المتزايدة لكافة العلوم في عصرنا الحاضر، الذي يمتاز بتنامي المعرفة الإنسانية والإنتاج الفكري والعلمي للبشرية، وما صاحب ذلكي ذلك من تقدم تكنولوجي مذهل وثورة معلوماتية هائلة،

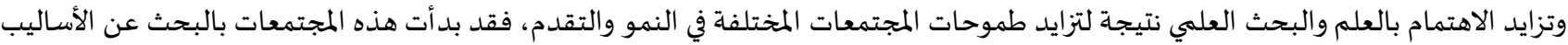
العلمية لإيجاد الحلول لمشكلاتها؛ فكان أن انتشرت مراكز البحث العلمي وأصبح الإنسان العادي يحتاج إلى التفكير العلمي في مواجهة مشكلاته،

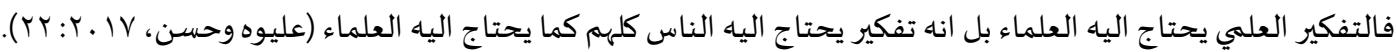


هذا الاهتمام بالعلم أدى إلى نشوء ثورة تكنولوجية تعتمد على المعرفة العلمياة، والاستخدام الأمثل للمعلومات المتدفقة بوتيرة سريعة، مما أدى المى

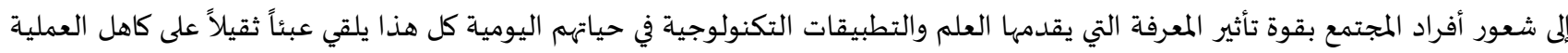
التعليمياة مما يجعل من متطلبات مجتمع القرن الحادي والعشرين بحاجة إلى معلم قادر على استيعاب منجزات الثورة العلمية والتكنولوجية، ومسلح

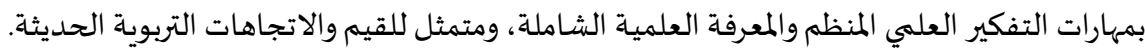

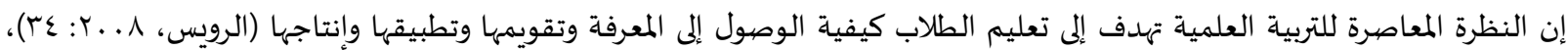
وحيث أن الكثير من المختصين في التربية العلمية يؤكدون أن الهدف الأساسي في تدريس العلوم هو : تعليم الطلبة كيف يفكرون، وكيف يضبطونه

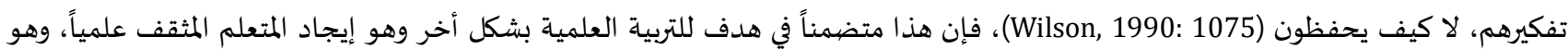

الهدف الأساسي في التربية العلمية (Deng, 2007: 11)، وإيجاد المتعلم الواعي لمفاهيم الثقافة العلمية والاستقصاء العلمي وطبيعة العلم. تعد طبيعة العلم وبنيته ركنا أساسيا وحجر الزاوية في التربية العلمية، وأصلا في تدريس العلوم ما يشهده من اهتمام واسع وتطوير مستمر في

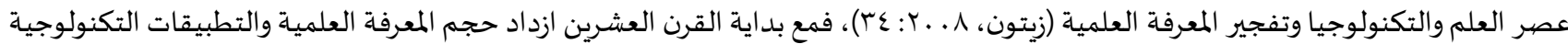

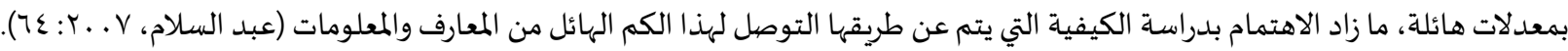
ونظراً لأهمية موضهوع فهم أبعاد الثقافة العلمية من جهة والاهتمام بالاستقصاء العلمي من جهة أخرى فقد تزايدت الدراسـات التربوية التي تناولت الثقافة العلمية، بعد أن أدرجت كثير من الدول ضيمن أهماد أهدافها لتدريس العلوم إكسـاب الطلاب مفاهيم الثقافة العلمياة، ومنها طبيعة العلم،

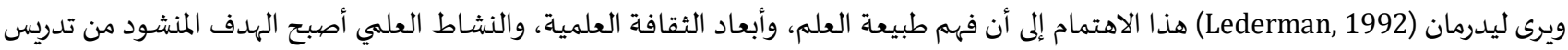

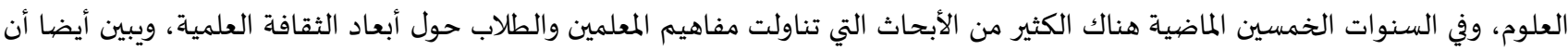

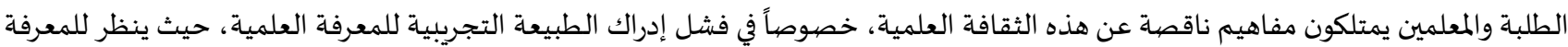
العلمياة في أغلب الأحيان كأها حقائق مطلقة حول العالم. وتبرز أهمية الثقافة العلمية على المستويين: الفردي والاجتماعي، فعلى المستوى الفردي فإنها تمنح الأفراد الثقة للاستجابة الذكية للأفعال، لهابل والأحداث التي تحدث في الطبيعة، ومراقبة البيئة الشخصية لهم، وعلى المستوى الاجتماعي تعد ضرورية لجميع المواطنين لدعم قيادة هذا الكوكب المبك

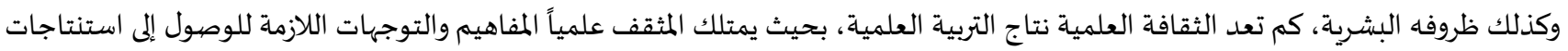
منطقية ذات معنى تساعده في اتخاذ قرارات سليمة (Aikenhead, 2006: 87). ويتجلى التركيز على هذا الهدف في العديد من المؤسسات والمنظمات الدولية بتحسين نوعية التربية العلمية لإيجاد مواطنين مثقفين علميا،

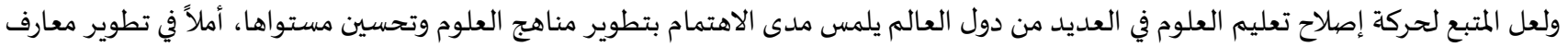
وسلوكيات الطلبة نحو امتلاك ثقافة علمية تؤهلهم للعيش في عصر المعلومات المتطور باستمرار، وتكتسب الثقافة العلمية أهميتها من المبادئ التي تنطلق وهي: تدريس العلوم بشكل أساسي لتطوير الاهتمام والوعي بالثقافة، والتركيز على التكنولوجيا كهدف أساسي، وتطوير ثقافة اجتماعية

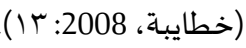

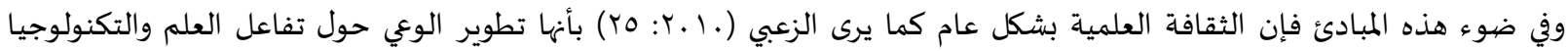

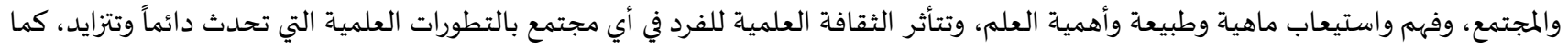

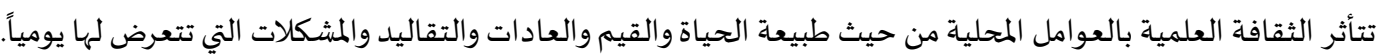

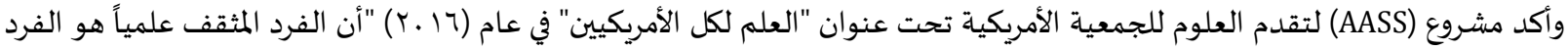

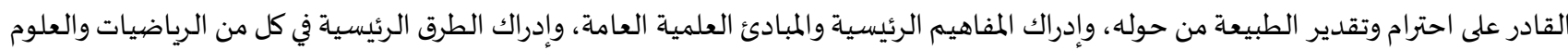
والتقنية ومدى اعتماد كل منها على الأخر لتحقيق التقدم، ومعرفة أن العلوم والرياضيات والتقنية تكوّن الجانب العلمي أو الأدائي لما يحيط باه من

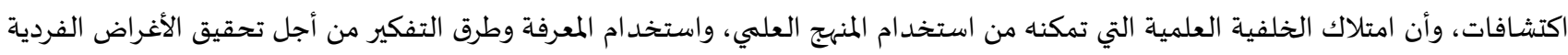

وتعتبر الثقافة العلمية من أهم الأهداف العامة لتدريس العلوم، وما يحدث في تدريس العلوم يتمثل في تركيز معلمي العلوم على بعد واحد من

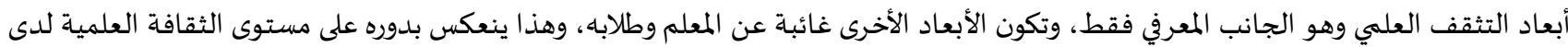

أفراد المجتمع ويحول دون تحقيق هدف رئيس من أهداف تدريس العلوم (الشهراني، . ... ؟: آب).

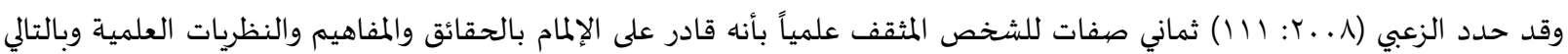
تطبيقها في حياته اليومياة، وإدراك طبيعة العلم، وامتلاك اتجاهات إيجابية نحو العلم والتكنولوجيا، وتقدير قيمة العلم والتكنولوجيا في المجتمع ومعرفة كيفية تأثير كل منهما في الأخر، ويمتلك القدرة على استخدام البحث العلمي في حل المشكلات، والقدرة على على اتخاذ القرارات الماتهات المستندة إلى قاعدة علمية في القضايا الاجتماعية، واستخدام المهارات العلمياة، وفهم البيئة من حوله. 
وبالتالي يهدف تدريس العلوم إلى إكساب الطلبة مهارات التفكير العلمي وأساليب البحث والاستقصاء العلمي، مما يساعدهم على حل ما

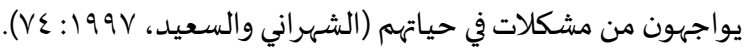

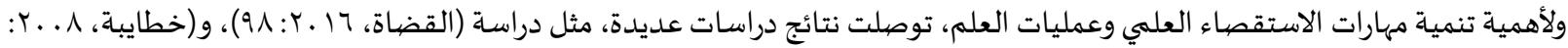

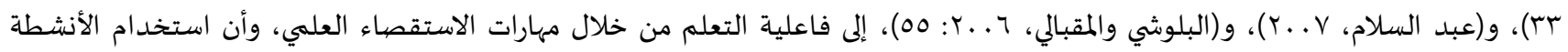

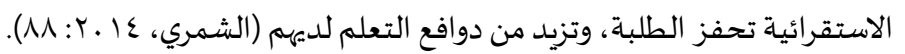

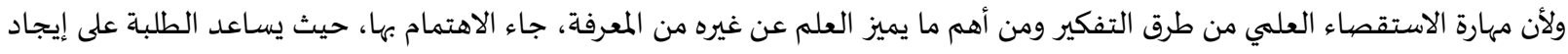

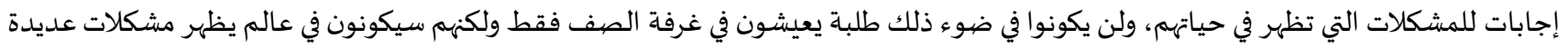

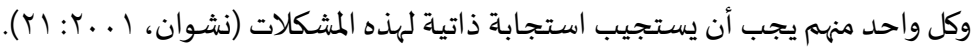

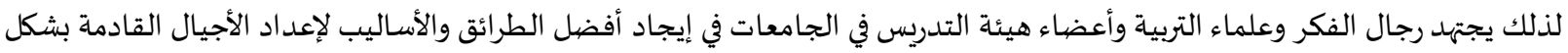

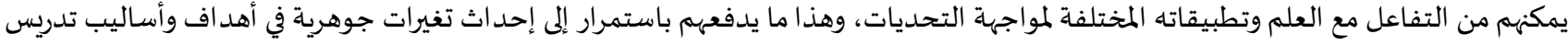

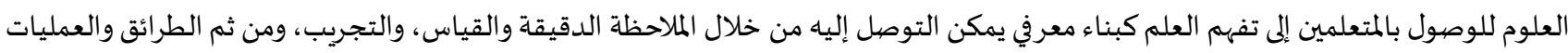

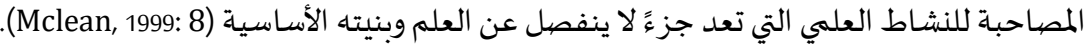

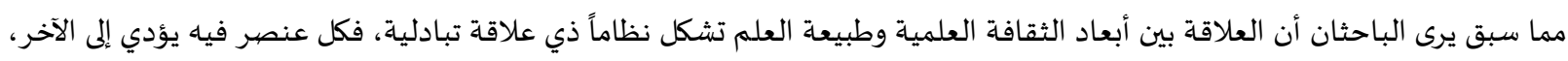

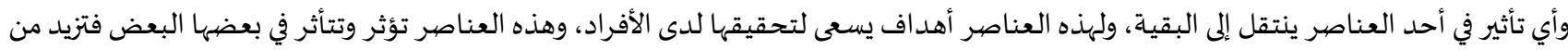

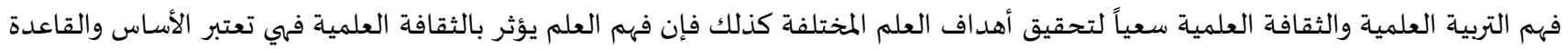
التي يبني علهيا الطلبة مفاهيمهم.

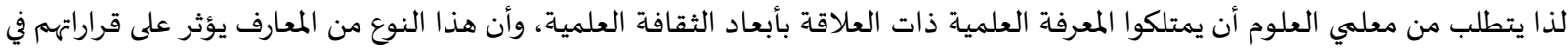

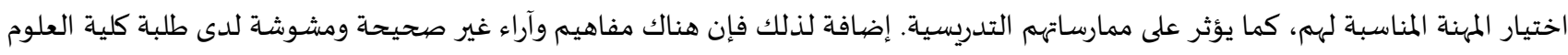

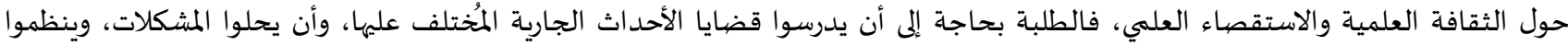

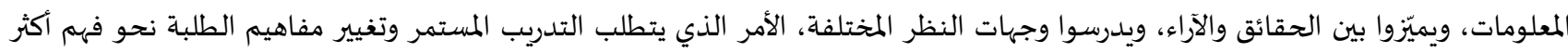

لأبعاد الثقافة العلمية وطبيعة العلم.

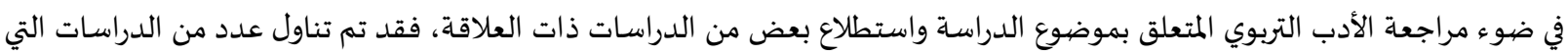

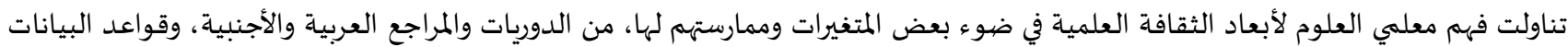

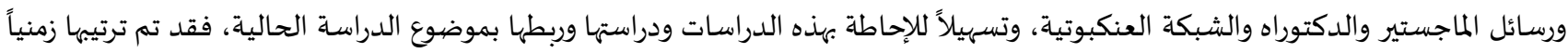

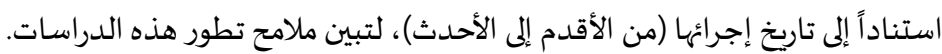

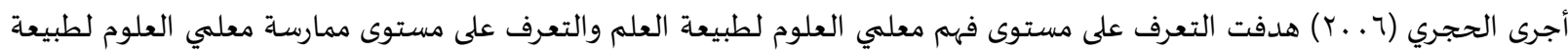

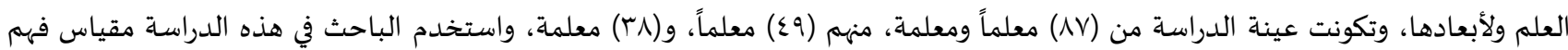

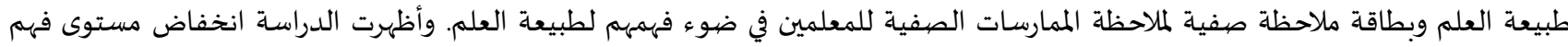

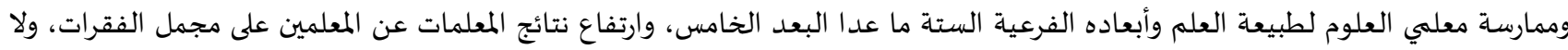
يختلف مستوى فهم طبيعة العلم باختلاف الخبرة التدريسية.

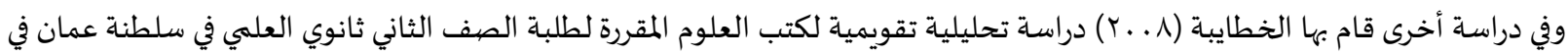

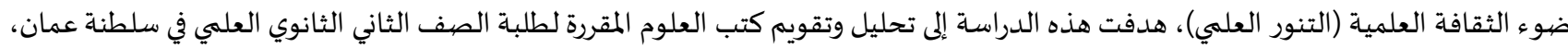

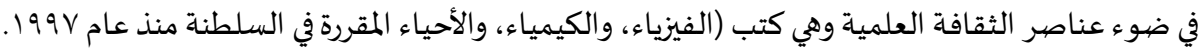

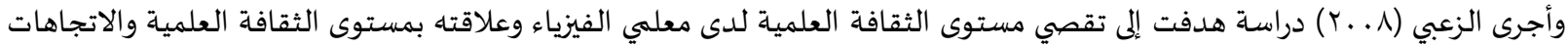

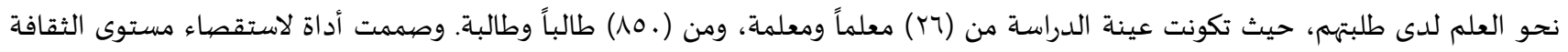

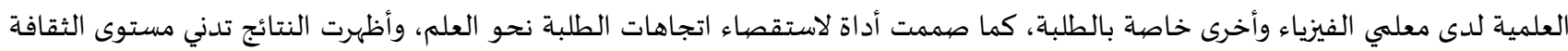

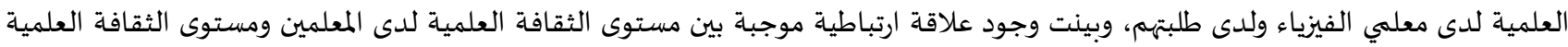
والاتجاهات نحو العلم لدى طلبتهم. وأجرى دلول (rا .ب) دراسة هدفت إلى التعرف على مستوى معلمي العلوم في مدارس غزة لطبيعة العلم والاستقصاء العلمي وفقا للمعايير

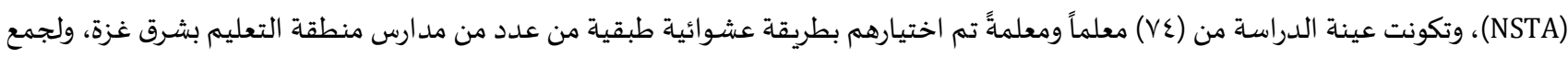

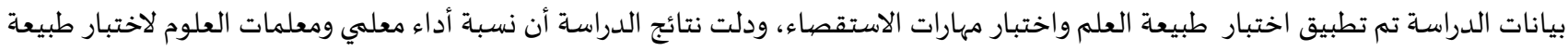


العلم واختبار مهارات الاستقصاء أقل من المقبول تربويا (.ی/)، كما أظهرت النتائج أن مستوى فهم طبيعة العلم يختلف باختلاف الجنس لصالح المعلمات.

وأجرى القضياة (7 ا ـ Y) دراسـة هدفت إلى تقصى مستوى فهم معلمي العلوم لطبيعة العلم وفق معايير الجمعية الوطنية لمعلمي العلوم (NSTA)

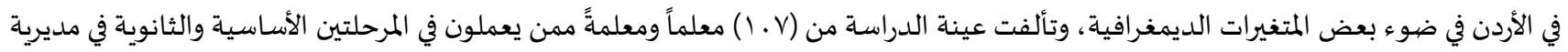

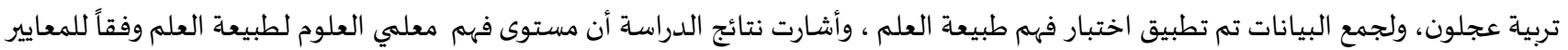
(NSTA)

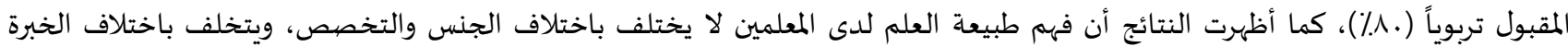

وأجرى كلاً من الرحيلي وسلوم (V ا • (Y) دراسة هدفت إلى قياس درجة الخيال العلمي لدى طالبات الصف الثاني ثانوي علمي المرتبط بوحدة الضوء في مادة الفيزياء، حيث تم استخدام المنهج الوصفي، حيث تم تحديد البعد الأول في القدرة على تقديم تصورات مستقبلية، وحلول لمواقف تتعلق

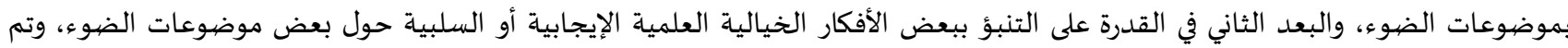
صياغة الأسئلة في صورة تساؤلات تدور حول موقف معين مرتبط بموضوعات وحدة الضوء، ويطلب من الطلبة الإجابة عنها بتقديم أكبر قدر ممكن من

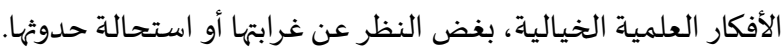

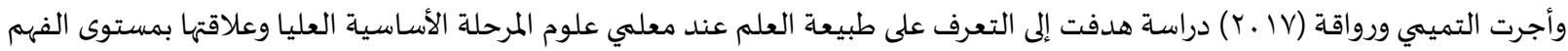

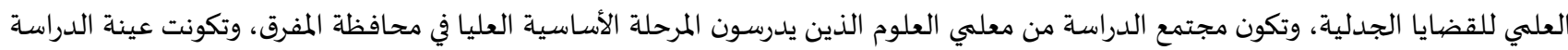

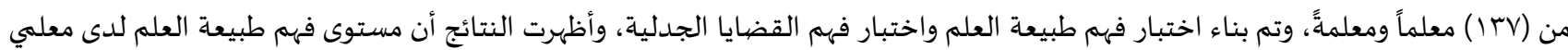
العلوم كان متوسطا، ولا يوجد أثر في فهم طبيعة العلم وفهم القضايا الجدلية، ووجود علاقة ارتباطية إيجابية بين فهم طبيعة العلم وفهم القضايا

يتضح من الدراسـات السابقة أن تقصي فهم أبعاد الثقافة العلمية، من المواضيع التي لم تحظى بالبحث مجتمعة - في حدود علم الباحثان-

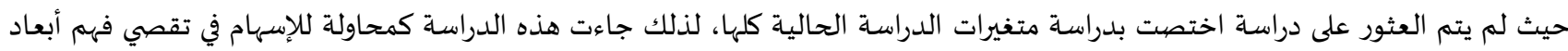

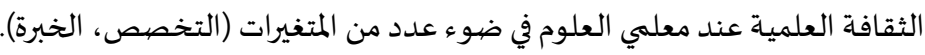

الطريقة والإجراءات:

وقد جاءت هذه الدراسة لاستقصاء فهم معلمي العلوم لأبعاد الثقافة العلمية وممارستهم لها، في ضوء بعض المتغيرات.

مشكلة الدراسـة وأسئلتها: أصبح العلم وتقنياته من الأمور اللازمة والضرورية لحياة كل فرد ليصبح مواطناً يساير عصره، ويجب على مؤسسات التعليم أن تساعد المتعلم

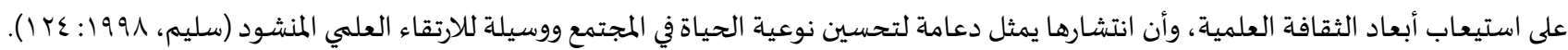
وقد أصبح نشر الثقافة العلمية متوفراً بين أيدي الطلبة من خلال مصادر مختلفة أهمها شبكات الاتصال ووسائلها، مما لها أثر في تعرض

المعلم لأسئلة تفوق مستوى الطلبة، وهم يرون معلمههم المصدرر الأساسي للمعرفة.

ومن هنا تأتي أهمية دور المعلم باعتباره المحرك الرئيس للعملية التعليمية التعلمية، إذ يعتبر المعلم مفكراً وقائداً للتعلم وباعثاً للثقافة العلمية"، وهذا يؤكد أن معلم العلوم مسؤول عن تنمية الثقافة العلمية لدى طلبته، وهذه المسؤولية تحتم عليه أن يكون ملماً بأبعاد الثقافة العلمية

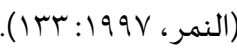

وفي حدود علم الباحثان لم تظهر في الأردن دراسات اهتمت بشكل شمولي بأبعاد الثقافة العلمياة، مما يدعو إلى ظهور دراسـة تضاف إلى الجهود

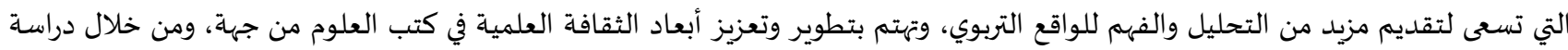
معارف المعلمين وممارساتهم لها في هذا المجال، وتتمثل مشكلة الدراسة بالسؤال الرئيسي: ما مستوى فهم معلمي العلوم لأبعاد الثقافة العلمية وممارستهم لها في ضوء بعض المتغيرات؟

وينبثق عن السؤال الرئيسي الأسئلة الفرعية الآتية:

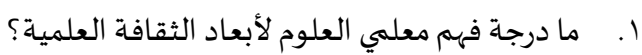

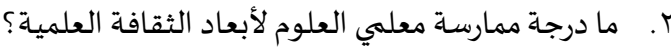

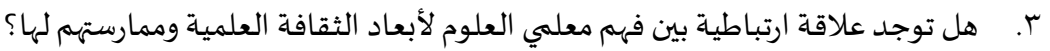

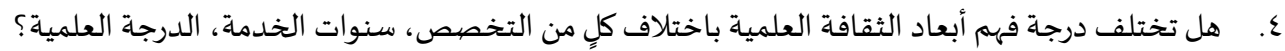

0. هل تختلف درجة ممارسة أبعاد الثقافة العلمية باختلاف كلٍ من التخصص، سنوات الخدمة، الدرجة العلمية؟ 
يتمثل الهدف الرئيسي من الدراسة في التعرف على مستوى فهم معلمي العلوم لأبعاد الثقافة العلمية وممارستهم لها في ضوء بعض المتغيرات

وينبثق مناه أهد اف فرعية كالآتي:

ا. التعرف على درجة فهم معلمي العلوم لأبعاد الثقافة العلمية.

r. التعرف على درجة ممارسة معلمي العلوم لأبعاد الثقافة العلمية.

r. التعرف على العلاقة بين فهم معلمي العلوم لأبعاد الثقافة العلمية وممارستهم لها.

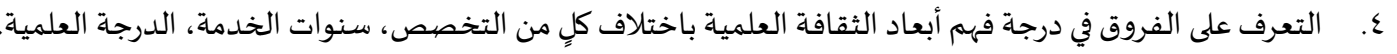
0. التعرف على الفروق في درجة ممارسة أبعاد الثقافة العلمية باختلاف كلٍ من التخصص، سنوات الخدمة، الدرجة العلمياة.

أهمية الدراسة ومبرراتها:

تتمثل أهمية الدراسة في المنحنيين النظري والتطبيقي:

الأهمية النظرية:

تكمن أهمية هذه الدراسة في تناولها لأكثر المفاهيم شيوعاً في التربية العلمية، ألا وهو أبعاد الثقافة العلمية. وتتناول هذه المفاهيم جانباً مهماً

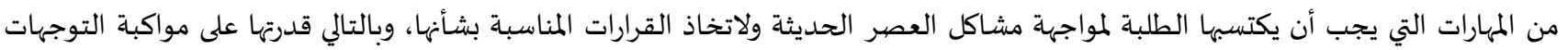
العالمياة، والأهد اف التربوية الحديثة المتعلقة بأبعاد الثقافة العلمية وطبيعة العلم، وبالتالي فإن زيادة الثقافة العلمية عند الأفراد، تمكن الفرد أن يكون

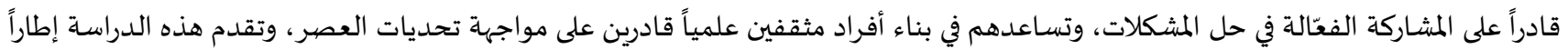
نظرياً في التعرف على مدى فهم معلمي العلوم لأبعاد الثقافة العلمية وطبيعة العلم، وهذه المعلومات تساعد القائمين على برامج إعداد المعلمين

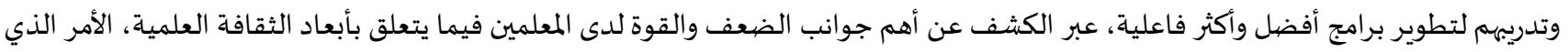
قد يسهم في تطوير أدائهم وتحسين كفايات طلبتهم .

الأهمية العملية:

يمكن أن تساعد هذه الدراسة المشرفيين التربويين في إعداد البرامج التدريبية والتطويرية المناسبة لمعلمي العلوم، للاتقاء بمستوى فهمهم

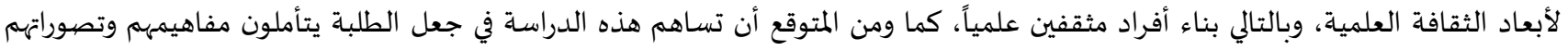

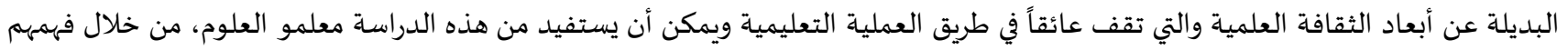

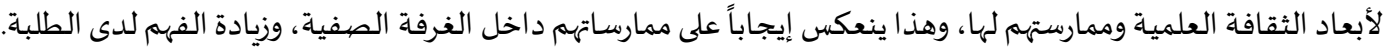
مصطلحات الدراسة وتعريفاتها الإجر ائية: الفهم: مهارة عقلية معرفية تتضمن القدرة على امتلاك معلمي العلوم لأبعاد الثقافة العلمية وطبيعة العلم، وتمثله في بنائه المعرفي، بحيث يستطيع أن

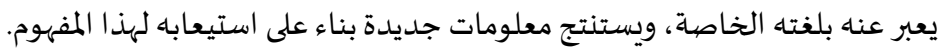
الثقافة العلمية: مجموعة المعارف والاتجاهات والمهارات التي يمتلكها الفرد في العلوم ويستطيع توظيفها في حياته اليومية، وتشكل هذه المعارف

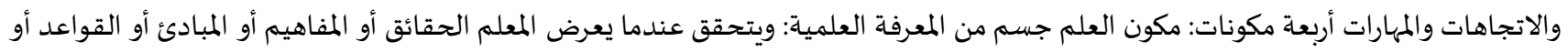

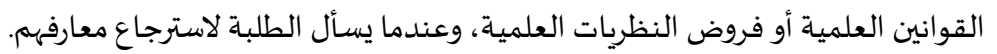

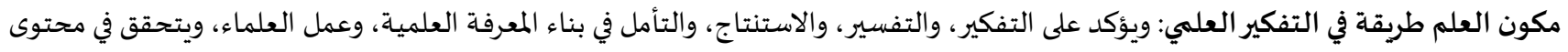

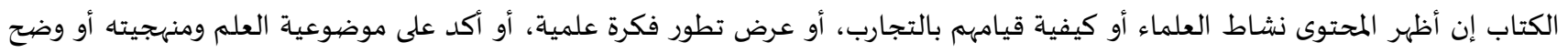

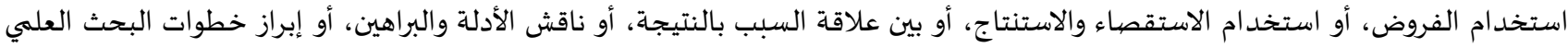

وحل المشكلات. مكون العلم طريقة في البحث والاستقصياء: ويتحقق في الثقافة العلمية إن وطلب من الطالب استخدام أساليب وعمليات العلم كالملاحظة والقياس

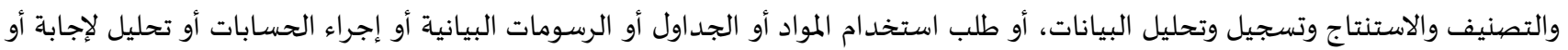
عمل تجربة أو القيام بنشاط أو مشروع علمي، وإجراء التواصل العلمي عبر كتابة التقارير أو التحدث الشفوي أو عرض مرئي باستخدام شرائح وجداول ورسومات بيانية ومخططات مفاهيمياة.

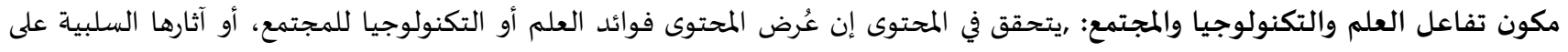

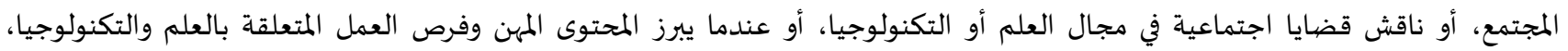

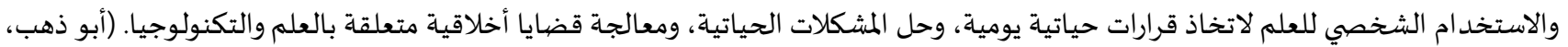

. (9:Y.. ₹ 
درجة الممارسة: مستوى أداء معلمي العلوم " الممارسة العملية- الجانب المهاري" لأبعاد الثقافة العلمية في الغرفة الصفية، والممارسة تتمثل بمجموعة من المعتقدات والتصورات أو التعليمات والقيم التي تتضمن تصوراً حول المعرفة وحول العلاقات بين الفرد والمجتمع في الممارسة.

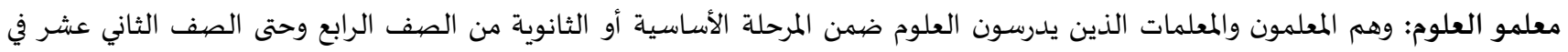

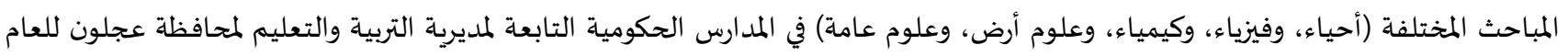

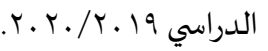

\section{حدود الدراسـة ومحددداتها:}

الحد الموضوعي: تناولت الدراسة فهم معلمي العلوم لأبعاد الثقافة العلمية في ضوء بعضاء بعض المتغيرات (التخصص، الخبرة) في محافظة عجلون. الحد المكاني: تم تطبيقها على معلمي العلوم في محافظة عجلون.

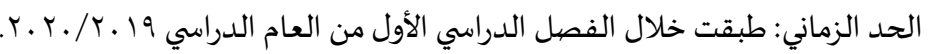

•المحددات المتعلقة بأداة الدراسة: دقة إجابات معلمي العلوم على أدوات الدراسة المستخدمة، وثبات أدوات الدراسة وصهدقها، ودرجة تمثيل العينة

منهج الدراسـة: تم استخدام المنهج الوصفي بأسلوب المستح، وذلك لملاءمته لطبيعة وأهداف الدراسـة.

مجتمع الدراسة وعينتها: تكون مجتمع الدراسة من جميع معلمي العلوم في مديرية تربية عجلون في التخصصيات: الفيزياء، والكيمياء، وعلوم الأرض، والأحياء، والعلوم

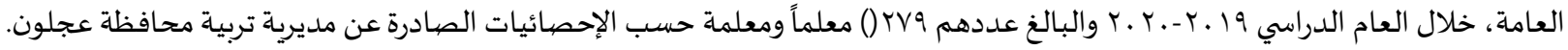

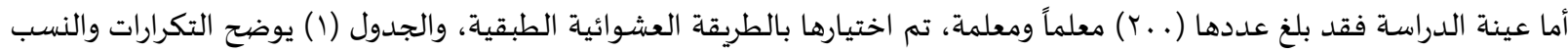

المئوية حسب متغيرات الدراسة.

جدول (1): التكرارات والنسب المئوية حسب متغيرات الدراسة

\begin{tabular}{|c|c|c|c|}
\hline النسبة & التكرار & الفئات & المتغير \\
\hline$r \varepsilon_{,}$. & $\varepsilon \wedge$ & فيزياء & التخصص \\
\hline$r \Lambda,$. & 07 & كيمياء & \\
\hline$r r,$. & $\varepsilon 7$ & أحياء & \\
\hline 17,0 & $r$ & علوم ارض & \\
\hline$\Lambda, 0$ & IV & علوم عامة & \\
\hline$r_{., .}$ & $\varepsilon$. & $0-1$ & سنوات الخدمة \\
\hline$r r_{,}$. & $\varepsilon \varepsilon$ & $1 .-7$ & \\
\hline $1 \varepsilon$, & ru & $10-11$ & \\
\hline$\varepsilon \varepsilon$, & 11 & اكثر من 10 & \\
\hline 70,0 & $1 \pi$ & بكالوريوس & الدرجة العلمية \\
\hline$r \varepsilon, 0$ & 79 & بكالوريوس + دبلوم & \\
\hline $1 \ldots$, & $r .$. & المجموع & \\
\hline
\end{tabular}

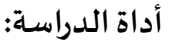

هدفت الدراسة لاستقصاء فهم معلمي العلوم لأبعاد الثقافة العلمية وممارستهم لها في ضوء بعض المتغيرات في مديرية تربية عجلون، ولتحقيق

أهد اف الدراسة تم بناء الأداة الآتية:

\section{أولاً: اختبارفهه معلمي العلوم لأبعاد الثقافة العلمية}

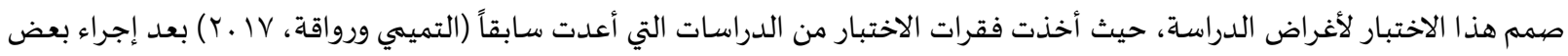

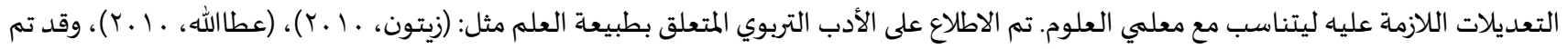

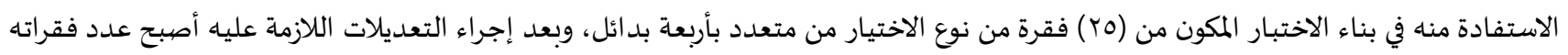
( (r. فقرة؛ حيث تم حذف عدد من الفقرات وإضافة فقرات جديدة، لتتناسب فقراته مع أهداف الدراسة ومعلمي العلوم، وأعطيت كل فقرة علامة واحدة، وقد توزعت الأسئلة على أربعة أبعاد (العلم طريقة التفكير، والعلم جسمر من المعرفة، والعلم طريقة في الاستقصياء، وتفاعل العلم والتكنولوجيا

والمجتمع). 
صدق الاختبار:

قام الباحثان للتحقق من صداق الاختبار من خلال عرضها على لجنة تحكيم من التربويين المتخصصيين في مناهج العلوم وأساليب تدريسها، لإبداء

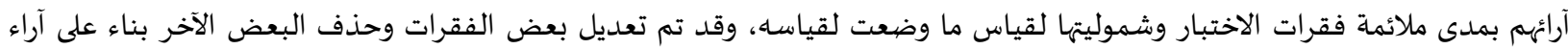
المحكمين وملاحظاتهم، وبلغ عدد فقرات الاختبار في صورته الهنائية (. (T) فقرة. ثبات الاختبار: للتأكد من ثبات أداة الدراسة، فقد تم التحقق بطريقة الاختبار وإعادة الاختبار (test-retest) بتطبيق الاختبار، وإعادة تطبيقه بعد أسبوعين

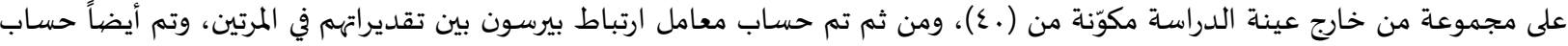

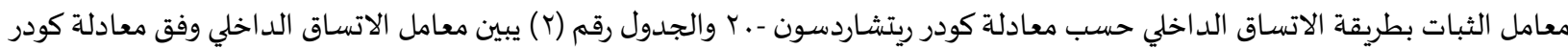

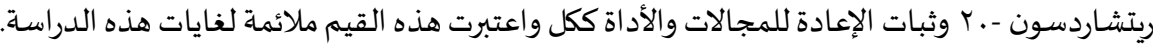

معامل الصعوبة والتمييز:

باستخدام برنامج (SPSS) تم تحليل استجابات عينة الدراسة لحساب معاملات الصعوبة والتمييز لفقرات الاختبار، حيث تم اعتماد النسبة

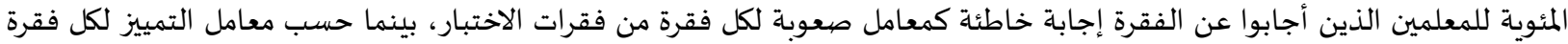

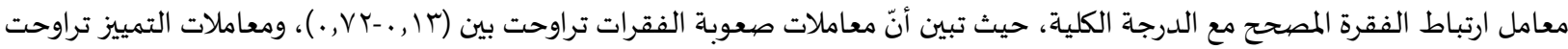

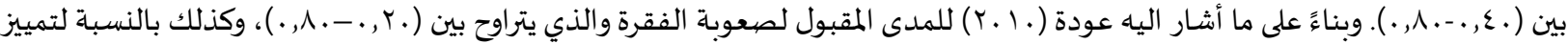

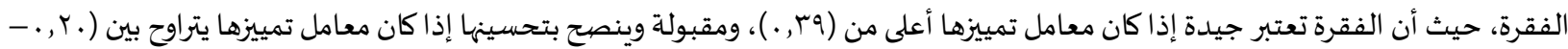

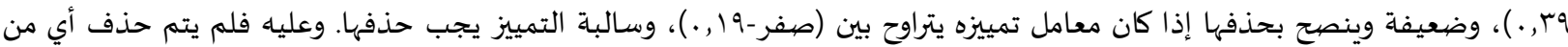
الفقرات بناء على معامل الصعوبة أو معامل التمييز. ثانياً: مقياس ممارسة معلمي العلوم لأبعاد الثقافة العلمية: قام الباحثان بالرجوع إلى الأدب النظري والدراسات السابقة، حيث تم أخذ فقرات الاستبانة من الدراسات التي أعدت سابقاً (الحجري، ج . . T) التي تم تطويرها وتعديلها بما يتناسب مع أغراض الدراسة.

• مبدق المقياس:

تم التحقق من صدق محتوى أداة الدراسة من خلال عرضها على لجنة تحكيم من التربويين المتخصصيين في مناهج العلوم وطرائق تدريسها، لإبداء

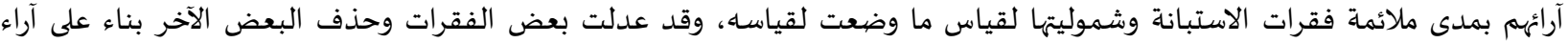
المحكمين وملاحظاتهم، وبلغ عدد فقرات الاستبانة في صورتها الهائية (rV) فقرة. ثبات المقياس:

للتأكد من ثبات المقياس، فقد تم التحقق بطريقة الاختبار وإعادة الاختبار (test-retest) بتطبيق المقياس، وإعادة تطبيقه بعد أسبوعين على إنى

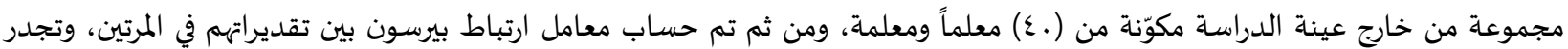

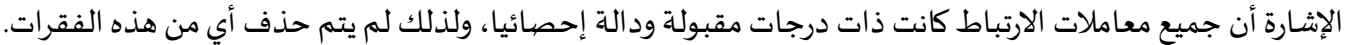

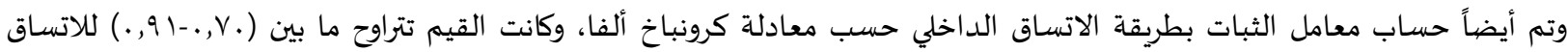
الداخلي، ومن (9/, .-- 9 , .) ككل واعتبرت هذه القيم ملائمة لغايات هذه الدراسة. متغيرات الدراسة: اشتملت الدراسة على المتغيرات الآتية:

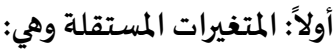
التخصص وله خمس فئات: فيزياء، كيمياء، أحياء، علوم أرض، علوم عامة. سنوات الخدمة ولها أربع فئات: ا-0 سنوات، 7-. 1 سنوات، 11 -10 سنة، أكثر من 10 سنه. • الدرجة العلمية وله فئتان: بكالوريوس، دبلوم عالي.

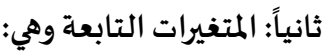
فهم معلمي العلوم لأبعاد الثقافة العلمية. ممارسة معلمي العلوم لأبعاد الثقافة العلمية. 
تم اعتماد سلم ليكرت الخماسي لتصحيح أدوات الدراسـة، بإعطاء كل فقرة من فقراته درجة واحدة من بين درجاته الخمس (موافق بشدة، موافق، محايد، معارض، معارض بشـة) وهي تمثل رقمياً (0، ع، ؟، ؟، ( ) على الترتيب، وقد تم اعتماد المقياس التالي لأغراض تحليل النتائج:

\begin{tabular}{|c|c|}
\hline قليلة & من . . 1- سז,r \\
\hline متوسطة & من ع r, \\
\hline كبيرة & $0, \ldots-r$ \\
\hline
\end{tabular}

وقد تم احتساب المقياس من خلال استخدام المعادلة التالية: (الحد الأعلى للمقياس - الحد الأدنى للمقياس) / عدد الفئات المطلوبة المعاه

$1, r+r=r /(1-0)$

ومن ثم إضافة الجواب (rس, (1) إلى نهاية كل فئة.

عرض ومناقشـة النتائج

أولاً: عرض ومناقشـة النتائج المتعلقة بسؤال الدراسة الأول: ما درجة فهم معلمي العلوم لأبعاد الثقافة العلمية؟ للإجابة عن هذا السؤال تم استخراج الأهمية النسبية لدرجة فهم معلمي العلوم لأبعاد الثقافة العلمية، والجدول (r) يوضح ذلك.

جدول (Y): المتوسطات الحسابية والانحر افات المعيارية والأهمية النسبية لدرجة فهم معلمي العلوم لأبعاد الثقافة العلمية مرتبة تنازلياً حسب الأهمية

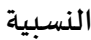

\begin{tabular}{|c|c|c|c|c|c|}
\hline الأهمية النسبة\%\% & الانحراف المعياري & المتوسط الحسابي & المجال & الرقم - ا & الرتبة \\
\hline vo & $1,17 r$ & $r, .$. & العلم طريقة التفكير & $r$ & 1 \\
\hline 01 & $r, 190$ & $0, r \varepsilon$ & العلم جسم من المعرفة & 1 & r \\
\hline$\infty 0$ & $T, I \varepsilon Y$ & $\mathrm{r}, \mathrm{AV}$ & العلم طريقة في الاستقصاء & $r$ & $r$ \\
\hline 01 & 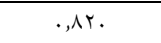 & $1, . r$ & تفاعل العلم والتكنولوجيا والمجتمع & $\varepsilon$ & $\varepsilon$ \\
\hline 7. & $0,7 Y \varepsilon$ & IT,IT & اختبار الثقافة العلمية & & \\
\hline
\end{tabular}

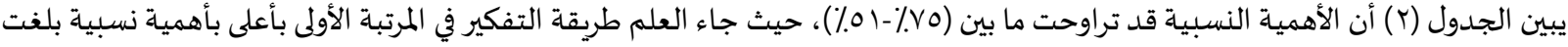

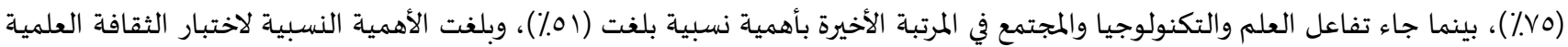

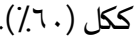

وقد يعزى ذلك إلى أن الثقافة العلمية من الأهد اف بعيدة المدى التي تحتاج وقت طويل، ولا يمكن تحقيقها من موضوع دراسي أو حصة دراسية واحدة أو حتى مقرر دراسي من مقررات العلوم، أيضاً ليست حكراً على معلمي العلوم، أنما تحتاج مشاركة من جميع المعلمين والمجتمع والمؤسسات التربوية لإعداد الطلبة واكسابهم الثقافة العلمية.

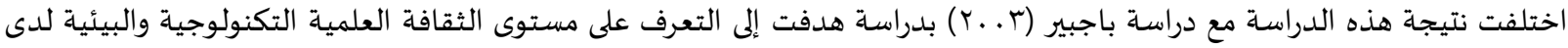
طلبة جامعة حضرموت للعلوم والتكنولوجيا ومصادر اكتسابهم لها، التي أشـارت إلى تدني مستوى الثقافة العلمية الكلي والذي بلغ م,عء \% مقارنة مع علامة المحك .0 0 0

كما واختلفت مع نتيجة دراسة صلاح ( . . ب) التي هدفت إلى قياس مستوى الجانب المعرفي للثقافة العلمية لدى طلبة الصفف الأول الثانوي في الأردن في ضوء متغيرات تعليمية، التي أشارت نتائج الدراسة أن مستوى الجانب المعرفي للثقافة العلمية لدى الطلبة أقل من علدية علامة المحك المحددد.

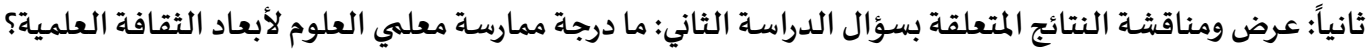

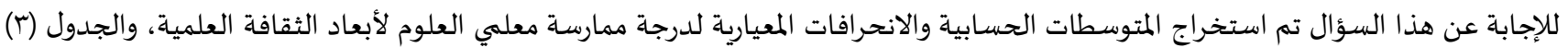
يوضح ذلك.

جدول (r): المتوسطات الحسابية والانحر افات المعيارية لدرجة ممارسة معلمي العلوم لأبعاد الثقافة العلمية مرتبة تنازلياً حسب المتوسطات الحسابية

\begin{tabular}{|c|c|c|c|c|c|}
\hline 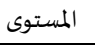 & الانحراف المعياري & المتوسط الحسابي & 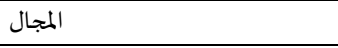 & 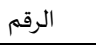 & 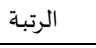 \\
\hline 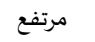 & ., $\{97$ & $r, q \vee$ & العلم جسم من المعرفة & 1 & 1 \\
\hline مرتفع &.,$\leqslant 7 V$ & $r, q r$ & العلم طريقة في الاستقصاء & $r$ & $r$ \\
\hline 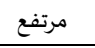 &., 0.4 & $r, \mathrm{VV}$ & العلم طريقة في التفكير & $\varepsilon$ & $r$ \\
\hline متوسط &., 07. & $r, 77$ & تفاعل العلم والتكنولوجيا والمجتمع & $r$ & $\varepsilon$ \\
\hline مرتفع &., 499 & $r, \lambda r$ & مقياس الثقافة العلمية & & \\
\hline
\end{tabular}




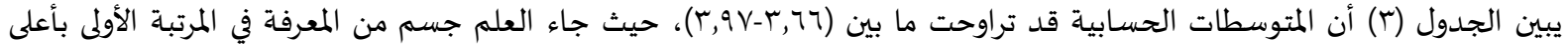

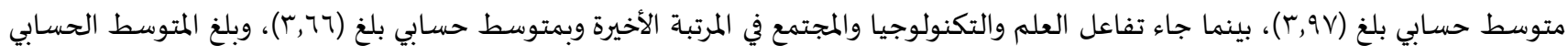

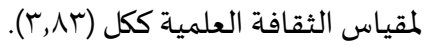

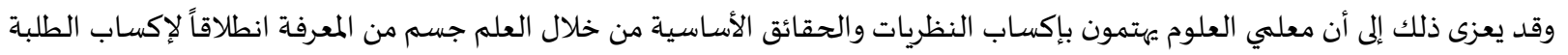

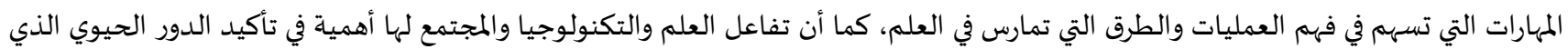
يقع على المعلم في نشر الثقافة العلمية.

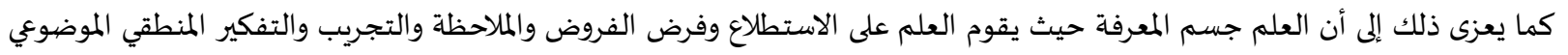

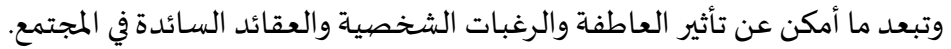

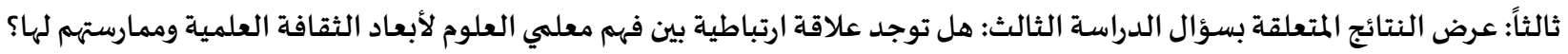

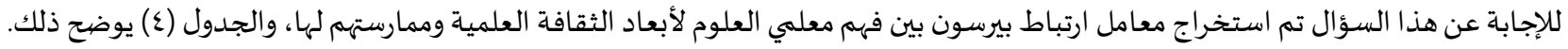

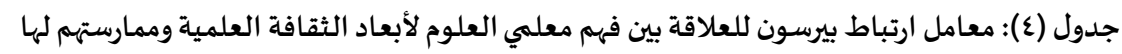

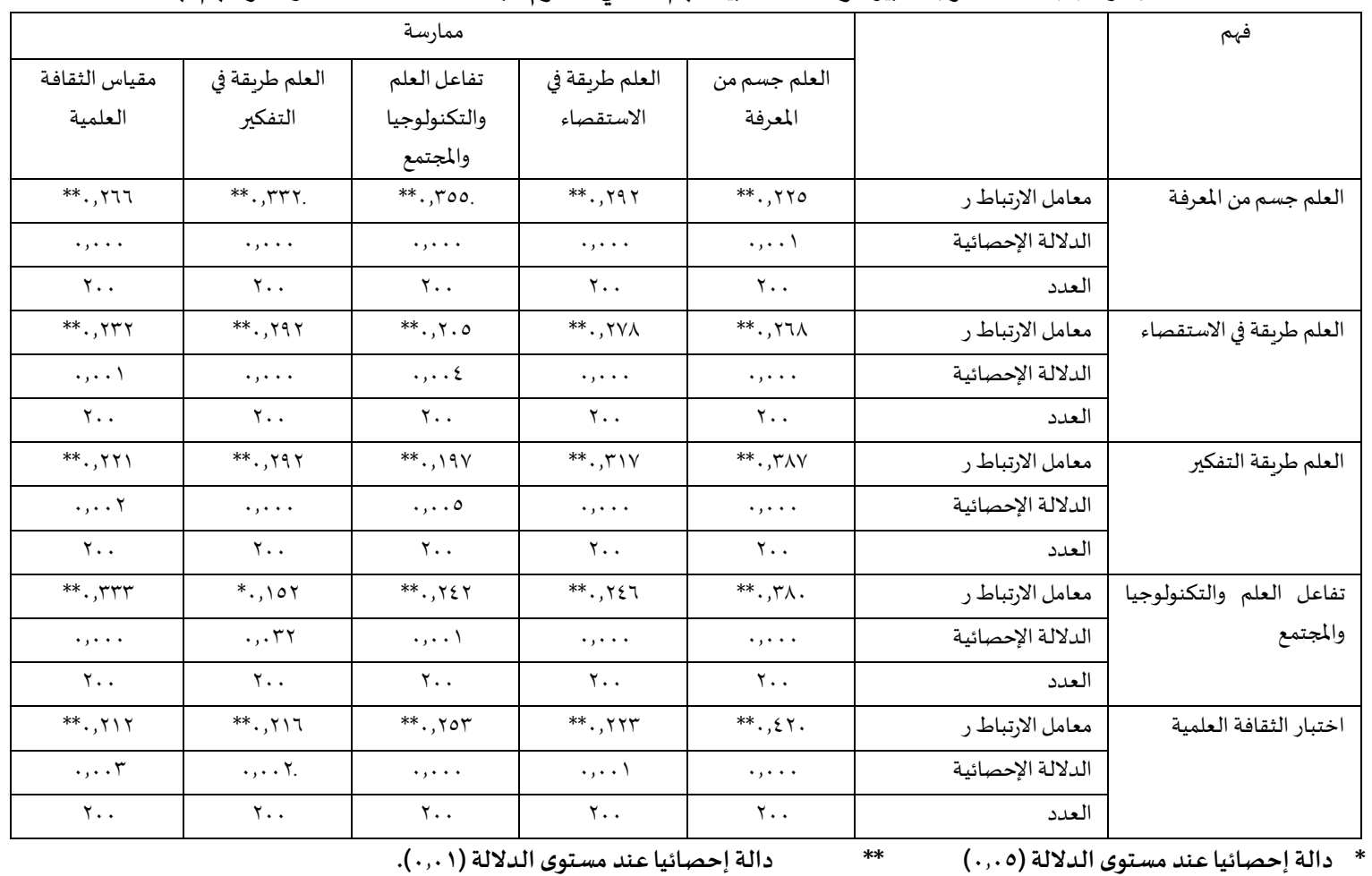

يتبين من الجدول (ع) وجود علاقة ايجابية دالة إحصائياً بين فهم معلمي العلوم لأبعاد الثقافة العلمية وممارستهم لها.

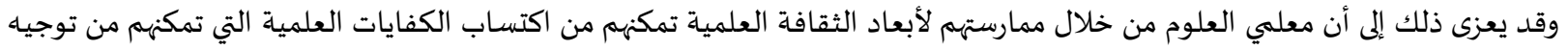

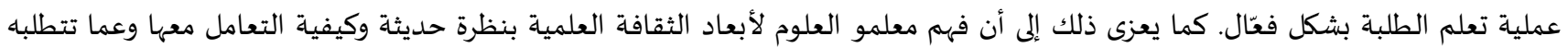
الأساليب المناسبة لتعلم العلوم وتعليمها.

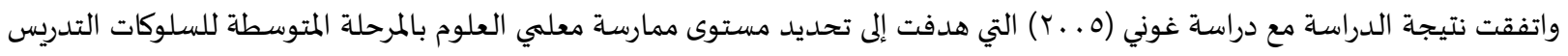
الاستقصائي العلمي، والتي أشارت نتائج الدراسة وأنه يوجد علاقة ارتباطية بين الخبرة التدريسية وممارسة معلمي العلوم بالمرحلة المتوسطة لسلوكيات التدريس الاستقصائي العلمي.

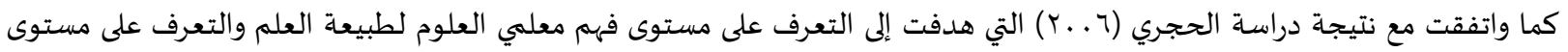

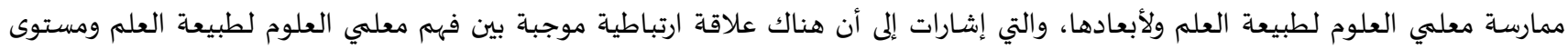
ممارسة معلمي العلوم لطبيعة العلمولأبعادها. رابعاً: عرض النتائج المتعلقة بسؤال الدراسة الر ابع: هل تختلف درجة فهم أبعاد الثقافة العلمية باختلاف كلٍ من التخصص، الخبرة؟ 
للإجابة عن هذا السؤال تم استخراج المتوسطات الحسابية والانحرافات المعيارية لدرجة فهم أبعاد الثقافة العلمية حسب متغيرات التخصص والخبرة، والجدول (0) يوضح ذلك.

جدول (0): المتوسطات الحسابية والانحر افات المعيارية لدرجة فهم أبعاد الثقافة العلمية حسب متغيرات التخصص، والخبرة، والدرجة العلمية

\begin{tabular}{|c|c|c|c|c|c|c|c|}
\hline اختبار الثقافة & تفاعل العلم & التفلم طريقة & العلم طريقة في & العلم جسم من & & & \\
\hline $1 r, 7$. & 1,10 & $r, 91$ & $\varepsilon, 1 \pi$ & $0, r_{0}$ & س & \multirow[t]{2}{*}{ فيزياء } & التخصص \\
\hline $0, \varepsilon r q$ & . Aro & $1,1.1$ & $1, v 9 \varepsilon$ & r,qvq & $\varepsilon$ & & \\
\hline $1 T, . \varepsilon$ & $1, \ldots$ & $r, . \varepsilon$ & $r, 91$ & $0, .9$ & س & \multirow[t]{2}{*}{ كيمياء } & \\
\hline $0,0 \ldots$ &., 1.9 & $1, Y .7$ & $r, 1 T 7$ & r,Ar. & $\varepsilon$ & & \\
\hline $1 r, 70$ & $\cdot, \wedge 9$ & $r, 10$ & r,人 & $0, \mathrm{VA}$ & س & \multirow[t]{2}{*}{ احياء } & \\
\hline $0, V Y \varepsilon$ & - & 1,1 Tro & $r, 0$, & T,A T & $\varepsilon$ & & \\
\hline $1 r, v 9$ & .,9v & $r, . r$ & $r, q$ & $\varepsilon, 10$ & س & \multirow[t]{2}{*}{ علوم ارض } & \\
\hline $0, \wedge \Gamma_{0}$ & . & $1, . \varepsilon_{0}$ & T,1.7 & $r, 909$ & $\varepsilon$ & & \\
\hline $11, r 9$ & 1,11 & r,or & $r, q \varepsilon$ & $\varepsilon, 70$ & س & \multirow[t]{2}{*}{ علوم عامة } & \\
\hline $7,1.1$ & $\cdot, \mu \mu r$ & $1, \varepsilon 7 \pi$ & $r, . \varepsilon_{0}$ & $r, . . \varepsilon$. & $\varepsilon$ & & \\
\hline 11,10 & $\cdot, 70$ & $r, v$. & $r, \varepsilon \wedge$ & $\varepsilon, V_{0}$ & س & \multirow[t]{2}{*}{$0-1$} & الخبرة \\
\hline $7, . \wedge$. & ., $\mathrm{VHr}$ & 1,170 & T,YT. & $r, .17$ & $\varepsilon$ & & \\
\hline $1 \varepsilon, \Gamma$ & .,95 & $r, 17$ & $\varepsilon, \cdot V$ & $7, . \mathrm{V}$ & س & \multirow[t]{2}{*}{$1 .-7$} & \\
\hline $0, . Y$. & $\cdot, \wedge \leqslant 7$ & $1, \cdot \mathrm{VV}$ & r,IVT & r,V7o & $\varepsilon$ & & \\
\hline $1 ., 94$ & $1, . \mathrm{V}$ & $\mathrm{r}, \mathrm{OV}$ & $r, r r$ & $r, 97$ & س & \multirow[t]{2}{*}{$10-11$} & \\
\hline$\varepsilon, V \mu V$ & $\cdot, 101$ & $1, r r$. & $1, \wedge \varepsilon V$ & T,Y.Y & $\varepsilon$ & & \\
\hline 15,91 & $1, r(T$ & $r, r$. & $\varepsilon, 11$ & $0, \varepsilon \varepsilon$ & س & \multirow[t]{2}{*}{ اكثر من 10} & \\
\hline $0,7 \wedge \mathrm{V}$ & ., . & $1, . \wedge \varepsilon$ & $5,1.9$ & r,QTr & $\varepsilon$ & & \\
\hline Ir,Tr & $\cdot, \wedge 9$ & $r, \wedge \varepsilon$ & $r, 01$ & $0, .1$ & س & بكالوريوس & \multirow[t]{3}{*}{ الدرجة العلمية } \\
\hline 0,419 & $\cdot, 117$ & $1, T Y V$ & r..r. & $r, 910$ & $\varepsilon$ & & \\
\hline $1 \varepsilon, 70$ & 1,17 & r,Tr & $\varepsilon, \varepsilon 1$ & $0,7 V$ & س & \multirow[t]{2}{*}{ بكالوريوس + دبلوم } & \\
\hline $0,9 \cdot r$ & -, $\mathrm{V} \vee \mathrm{q}$ & - $974 \mathrm{r}$ & r, rON & T,ATV & $\varepsilon$ & & \\
\hline
\end{tabular}

يبين الجدول (0) تبايناً ظاهرياً في المتوسطات الحسابية والانحرافات المعيارية لدرجة فهم أبعاد الثقافة العلمية بسبب اختلاف فئات متغيرات

التخصص، والخبرة، والدرجة العلمية.

ولبيان دلالة الفروق الإحصائية بين المتوسطات الحسابية تم استخدام تحليل التباين الثلاثي المتعدد على المجالات جدول (7).

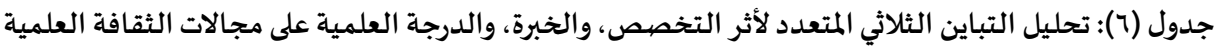

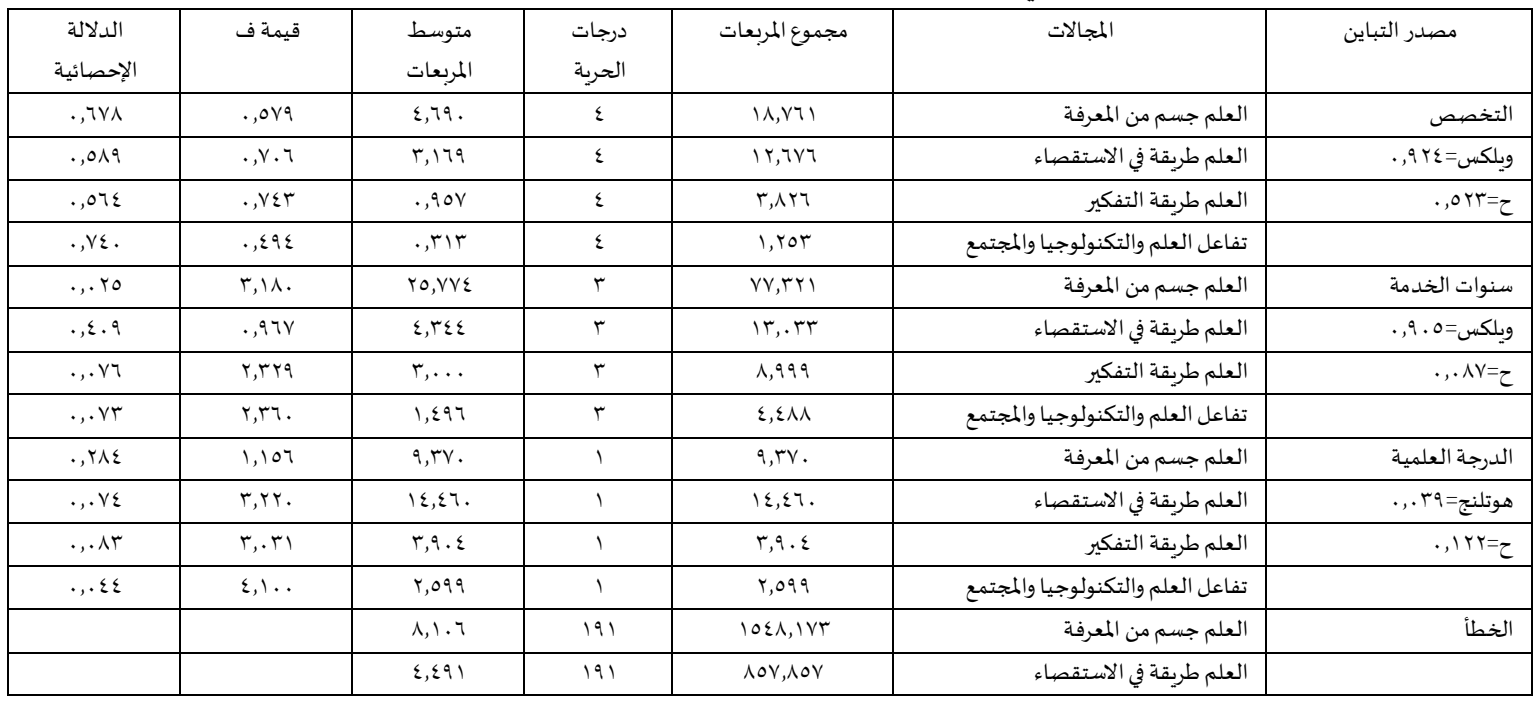




\begin{tabular}{|c|c|c|c|c|c|}
\hline & $1, r M M$ & 191 & $r\{0,991$ & العلم طريقة التفكير & \\
\hline & . & 191 & $|r|, . \wedge r$ & تفاعل العلموالتكنولوجيا والمجتمع & \\
\hline & & 199 & 1778,900 & العلم جسم من المعرفة & الكلي \\
\hline & & 199 & $91 r, 400$ & العلم طربقة في الاستقصاء & \\
\hline & & 199 & $r 71,990$ & العلمطريقة التفكير & \\
\hline & & 199 & $1 \% r, q 4$. & تفاعل العلم والتكنولوجيا والمجتمع & \\
\hline
\end{tabular}

يتبين من الجدول (7) الآتي:

عدم وجود فروق ذات دلالة إحصائية (م = 0. . . .) تعزى لأثر التخصص في جميع المجالات. عدم وجود فروق ذات دلالة إحصائية (م = 0 . . .) تعزى لأثر سنوات الخدمة في جميع المجالات.

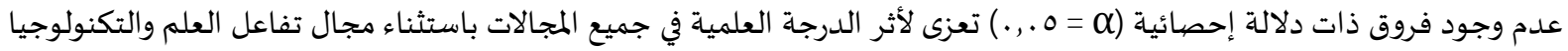
والمجتمع وجاءت الفروق لصالح بكالوريوس. وقد يعزى ذلك إلى أن جميع المعلمين على اختلاف تخصصياتهم أو سنوات خدمتهمه أو درجتهم العلمية مهتمون جميعا بفهم أبعاد الثقافة العلمية، وقد يعزى ذلك أيضا إلى أن جميع المعلمين يمرون بنفس الظروف المعين والدورات التدربيية المختلفة التي تطرحها وزارة التربية والتعليم أو أثناء مرحلتهم الدراسية مما أدى إلى ظهور هذه النتيجة.

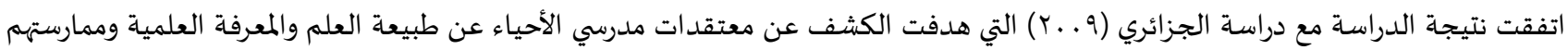
لها، والتي أشارت النتائج إلى عدم وجود فروق ذات دلالة تعود لمتغيري الخبرة التدريسية والمؤهل العلمي.

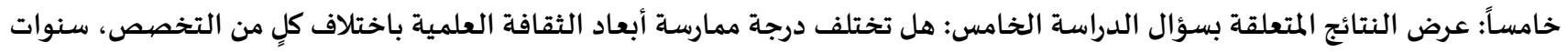
الخدمة، الدرجة العلمية؟ للإجابة عن هذا السؤال تم استخراج المتوسطات الحسابية والانحرافات المعيارية لدرجة ممارسة أبعاد الثقافة العلمية حسب متغيرات

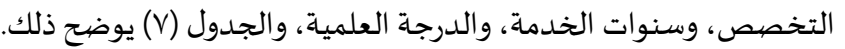

جدول (V): المتوسطات الحسابية والانحر افات المعيارية لدرجة ممارسة أبعاد الثقافة العلمية حسب متغيرات التخصص، والتهب والخبرة والدرجة العلمية

\begin{tabular}{|c|c|c|c|c|c|c|c|}
\hline مقياس الثقافة & التلم طريقة في & تواعل العلم & العلم طريقة في & العلم جسم من & & التخصص & \\
\hline r,Ar & r,vi & $r, 7 r$ & $r, q \varepsilon$ & $r, 90$ & س س & \multirow[t]{2}{*}{ فيزياء } & التخصص \\
\hline . Г人 &.,$\varepsilon V V$ &., 07 &.,$\leqslant 00$ &., $01 \mathrm{~V}$ & $\varepsilon$ & & \\
\hline r,vq & $r, v \varepsilon$ & $r, 7 \varepsilon$ & $r, \lambda r$ & $r, 97$ & س & \multirow[t]{2}{*}{ كيمياء } & \\
\hline . &., 001 &., 019 & . & ., $\leqslant \vee q$ & $\varepsilon$ & & \\
\hline$r, \Lambda V$ & $r, A r$ & r,VT & $r, 91$ & $r, 99$ & س س & \multirow[t]{2}{*}{ احياء } & \\
\hline . , זVר & .,OTY &., 01. & $\cdot, \varepsilon \wedge 10$ & .,OYT & $\varepsilon$ & & \\
\hline$r, \Lambda 1$ & $r, v \varepsilon$ & r,TV & $r, \wedge 9$ & $r, 91$ & س س & \multirow[t]{2}{*}{ علوم ارض } & \\
\hline . TVE & . rVq &., $0 \mathrm{VT}$ & $\cdot,\{7\}$ & $\cdot, \varepsilon V \varepsilon$ & $\varepsilon$ & & \\
\hline$r, q$. & r,vq & $r, 70$ & $\varepsilon, 11$ & $\varepsilon, . r$ & س س س & \multirow[t]{2}{*}{ علوم عامة } & \\
\hline$\cdot, \varepsilon \leqslant r$ &., $09 \mathrm{~V}$ & $\cdot, \varepsilon \cdot \wedge$ & . OTr &., 011 & $\varepsilon$ & & \\
\hline$r, \Lambda 0$ & $r, v r$ & $r, v i$ & $\varepsilon, \ldots$ & $\varepsilon, \cdot r$ & س س س & \multirow[t]{2}{*}{$0-1$} & الخبرة \\
\hline . rVq & ., $\{99$ & . &., $0 Y 1$ & ., $\{01$ & $\varepsilon$ & & \\
\hline r,vo & $r, v \varepsilon$ & $r, 71$ & $r, \lambda r$ & $r, \wedge \varepsilon$ & س س & \multirow[t]{2}{*}{$1 .-7$} & \\
\hline . rV. & ., $\varepsilon r$. &., 007 & . ז人т & .,00r & $\varepsilon$ & & \\
\hline$r, \Lambda \Lambda$ & $r, v 7$ & $r, 07$ & $\varepsilon, 1$. & $\varepsilon, 1 \Gamma$ & س س & \multirow[t]{2}{*}{$10-11$} & \\
\hline . &., 00. & .,OTr & $\cdot, \varepsilon \wedge 9$ & ., orv & $\varepsilon$ & & \\
\hline$r, \wedge \varepsilon$ & $r, \Lambda 1$ & $r, 79$ & $r, 19$ & $r, 9 v$ & س س & \multirow[t]{2}{*}{ اكثر من 10} & \\
\hline.,$\leqslant 10$ &., $0 Y 7$ & .,OYN &.,$\{7\}$ &.,$\{7\}$ & $\varepsilon$ & & \\
\hline r,vo & $r, 7 \Lambda$ & $r, 0 \varepsilon$ & $r, \Lambda \Lambda$ & $r, q r$ & س س & بكالوريوس & \multirow[t]{4}{*}{ الدرجة العلمية } \\
\hline ., rq1 & ., $\{\wedge 0$ &., 000 &., 0.1 &., 0.9 & $\varepsilon$ & & \\
\hline$r, 91$ & $r, 90$ & $r, \wedge 9$ & $\varepsilon, \cdot r$ & $\varepsilon, .7$ & س & \multirow[t]{2}{*}{ بكالوريوس + دبلوم } & \\
\hline , roג & $\cdot, \varepsilon \wedge \uparrow$ & $\cdot, \sum 99$ & $\cdot$, , & . & $\varepsilon$ & & \\
\hline
\end{tabular}

س= المتوسط الحسابي ع=الانحراف المعياري 
يبين الجدول (V) تبايناً ظاهرياً في المتوسطات الحسابية والانحرافات المعيارية لدرجة ممارسة أبعاد الثقافة العلمية بسبب اختلاف فئات متغيرات التخصص، وسنوات الخدمة، والدرجة العلمية.

ولبيان دلالة الفروق الإحصائية بين المتوسطات الحسابية تم استخدام تحليل التباين الثلاثي المتعدد على المجالات جدول (م).

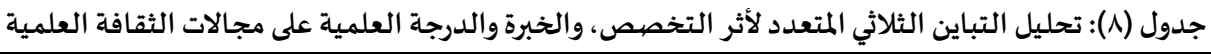

\begin{tabular}{|c|c|c|c|c|c|c|}
\hline الإحصائية الدلالة & ق قيمة ف & متوسط المربعات & درجات & مجموع المربعات & المجالات & مصدر التباين \\
\hline$\cdot, \wedge \vee 0$ & $\cdot, r \cdot \varepsilon$ & $\cdot, \cdot V r$ & $\varepsilon$ & . & العلم جسم من المعرفة & التخصص \\
\hline., $.1 \varepsilon$ & $r, r \cdot r$ & . & $\varepsilon$ & r,OYA & العلم طريقة في الاستقصاء & ويلكس=920. \\
\hline.,$V Y q$ &., 01 &., $1 \leqslant \wedge$ & $\varepsilon$ &., 091 & تفاعل العلم والتكنولوجيا والمجتمع & $.457=\tau$ \\
\hline.,$人 r q$ &., rov &.,.$\wedge \vee$ & $\varepsilon$ &.,$r \leqslant 7$ & العلم طريقة في التفكير & \\
\hline.,$\ldots \varepsilon$ & $\varepsilon, 0.9$ &.,$\wedge 9$ & r & r,TV. & العلم طريقة في الاستقصاء & ويلكس=888. \\
\hline., $\mathrm{rq}$. & 1, rov & 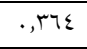 & $r$ & $1, .95$ & تفاعل العلم والتكنولوجيا والمجتمع & $.032=\tau$ \\
\hline., $9 Y \Lambda$ &., $10 r$ &.,.$M V$ & $r$ &., 111 & العلم طريقة في التفكير & \\
\hline.,.$+r 7$ & $\varepsilon, \varepsilon \vee$. & $1, . \vee \wedge$ & 1 & $1, \cdot v \wedge$ & العلم جسم من المعرفة & الدرجة العلمية \\
\hline \multirow{8}{*}{$\cdot, \ldots$} & &.,$T \leqslant 1$ & 191 & $\sum 7, .7 V$ & العلم جسم من المعرفة & 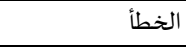 \\
\hline & &., $19 V$ & 191 & $r V, V \ldots$ & العلم طريقة في الاستقصاء & \\
\hline & &.,$Y q$. & 191 & $00, r \leqslant 9$ & تفاعل العلم والتكنولوجيا والمجتمع & \\
\hline & &.,$T \leqslant Y$ & 191 & $\sum 7, \zeta \wedge$. & العلم طريقة في التفكير & \\
\hline & & & 199 & $\varepsilon \wedge, \wedge १ ९$ & العلم جسم من المعرفة & 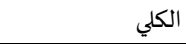 \\
\hline & & & 199 & $\varepsilon r, \varepsilon . \varepsilon$ & العلم طريقة في الاستقصاء & \\
\hline & & & 199 & 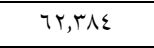 & تفاعل العلم والتكنولوجيا والمجتمع & \\
\hline & & & 199 & $0 ., 1 \leqslant Y$ & العلم طريقة في التفكير & \\
\hline
\end{tabular}

$$
\text { يتبين من الجدول (م) الآتي: }
$$

هدم وجود فروق ذات دلالة إحصائية (م = 0. . . ) تعزى لأثر التخصص في جميع المجالات. عدم وجود فروق ذات دلالة إحصائية (م = 0 . . .) تعزى لأثر سنوات الخدمة في جميع المجالات.

وجود فروق ذات دلالة إحصائية (م =0 . . .) تعزى لأثر الدرجة العلمية في جميع المجالات وجاءت الفروق لصالح بكالوريوس+دبلوم.

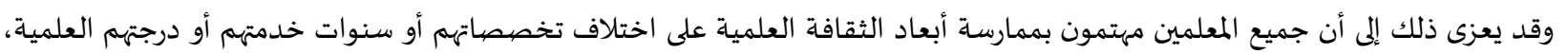
كما أههم تعرضوا خلال مراحلهم الدراسية إلى نفس المواقف التي تؤكد على أهمية ممارسة أبعاد الثقافة العلمية وفي ضوء فئه فلسفة وأهداف مشتركة، كما

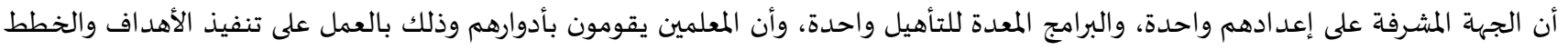

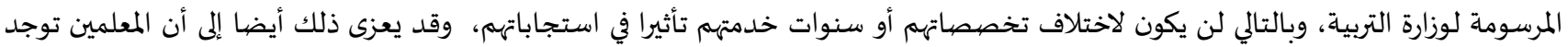

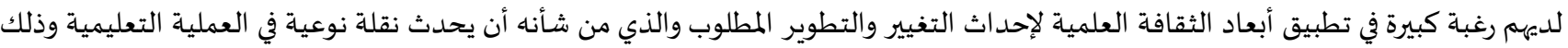
من خلال الخبرات والمعلومات التي تلقوها أثناء دراساتهم العليا.

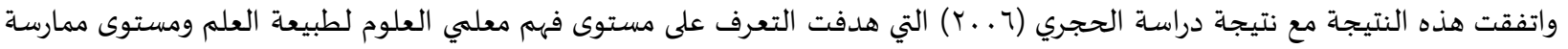
معلمي العلوم لطبيعة العلم ولأبعادها، والتي أشـارت نتائجها إلى أنه لا يختلف مستوى فهم طبيعة العلم باختلاف الخبرة التدريسية. التوصيات: في ضوء ما تقدم من عرض النتائج قام الباحثان بوضع مجموعة من التوصيات كالآتي:

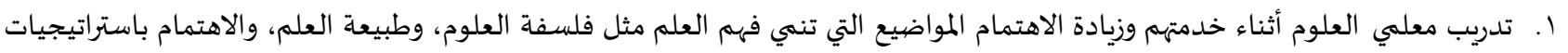
تدريسية حديثة تمكنهم من إبراز ذلك الفهم داخل الغرفة الصفية.

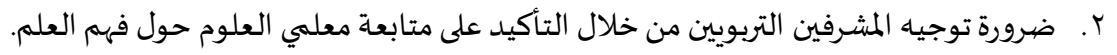

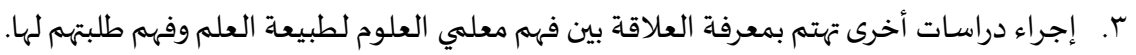
ع. إجراء المزيد من الدراسات التي تقوم بتحليل مناهج العلوم في المراحل التعليمية المختلفة. 


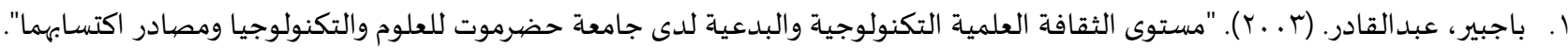
رسالة ماجستير غير منشورة. جامعة اليرموك. الاردن.

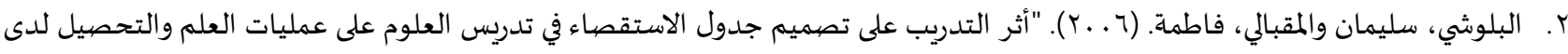

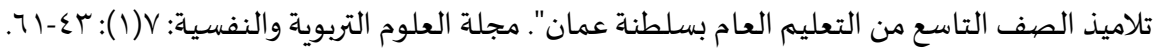

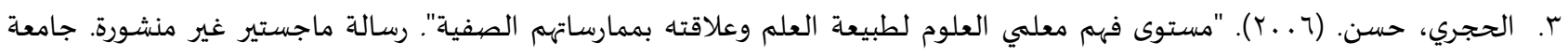
السلطان قابوس. سلطنة عمان.

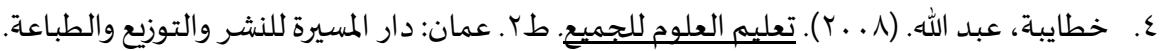

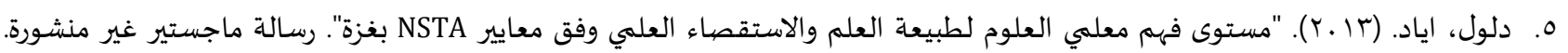
الجامعة الاسلامية. غزة.

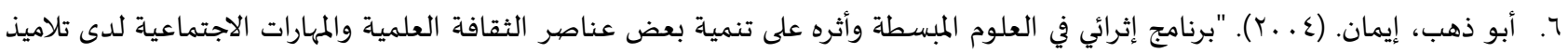

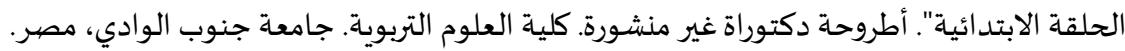

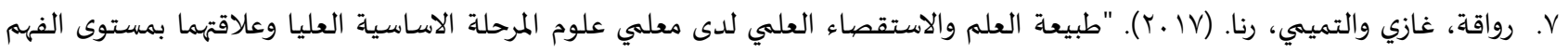

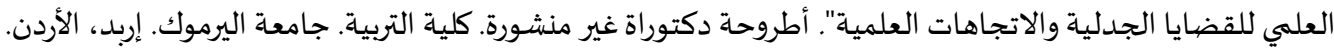

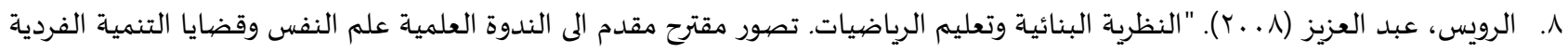

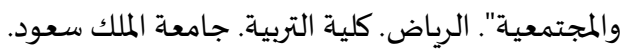

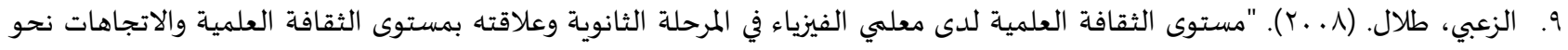

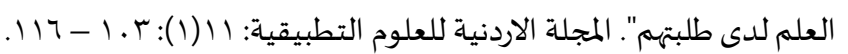

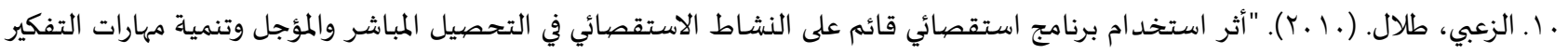

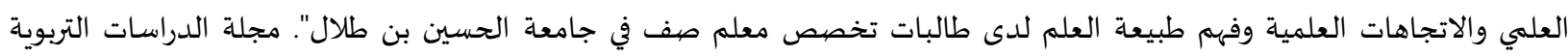

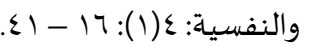

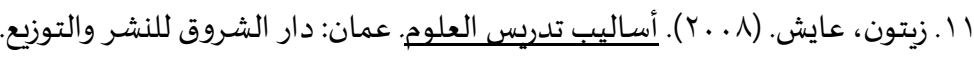

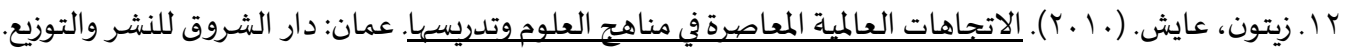

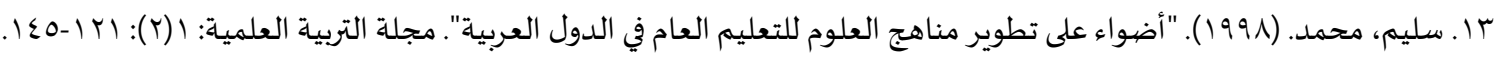

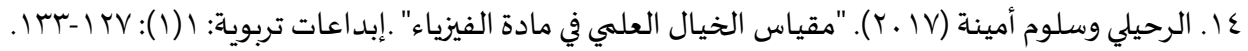

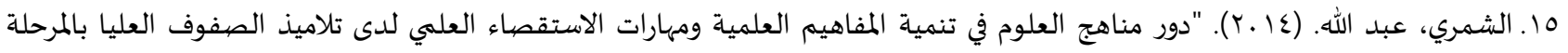

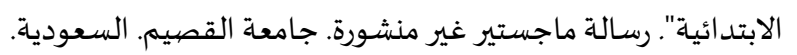

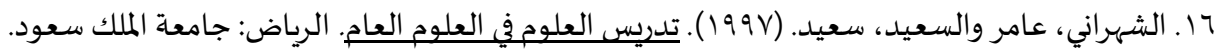

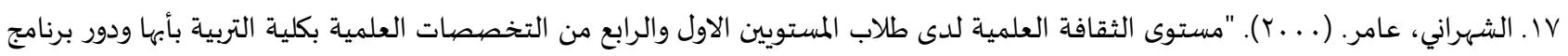

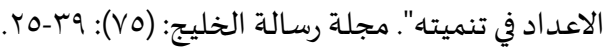

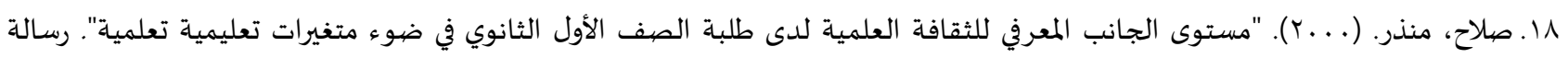
ماجستير غير منشورة. جامعة اليرموك. الاردن.

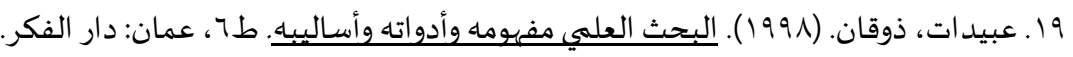

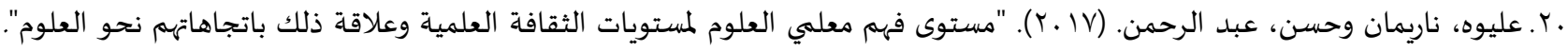

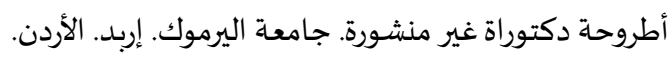

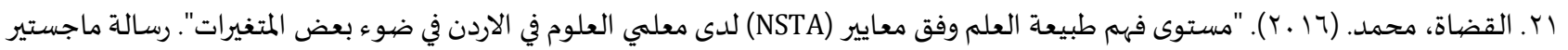
غير منشورة. جامعة آل البيت. المفرق، الأردن.

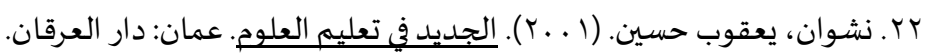
آז. النمر، مدحت (1997) ."فلسفة ومتطلبات اعداد معلم العلوم للقرن الحادي والعشرون". المؤتمر العلمي الأول، المجلد الاول، الإسكندرية. 
[1] AAAS. (1993). "Benchmarks for Science Literacy: Project 2061”. New York: Oxford University press.

[2] Aikenhead, S. (2006). "Science education for everyday- life: evidence- based practice". New York: Teachers College Press.

[3] American Association for the Advancement of Science (AAAS). (1990). Science for all Americans. New York: Oxford University press.

[4] Association for the Advancement of Science (AAAS). (1993). "Benchmarks for Science Literacy: A project 2061 Report". New York: Oxford University press.

[5] Chi, C. (2005). "First_YearPre_Service Teachers in Taiwan_ Do They enter the teacher program with Satisfactory scientific literacy and attitudes Toward?". International of Journal Science Education 27(13), 1549-1570, https://doi.org/10.1080/09585190500186401

[6] Deng, Z. (2007). "Scientific Literacy as an Issue of Curriculum Inquiry". Promoting Scientific Literacy: Science Education Research in Transaction Proceedings of the Linnaeus Tercentenary Symposium held at Uppsala University, Uppsala, Sweden, 21-25 March 2007.

[7] Lederman, G. (1992). "Students and teacher's conceptions of the nature of science: A re-assessment". School Science and Mathematics, 86, 91-99.

[8] Mclean, J. (1999). "Incorporating the use of Concept Maps and diagrams is student's formal lab report writing". Master thesis, Montma State University, USA.

[9] Murcia, K. (2006). "Scientific Literacy for Sustainability". PhD Thesis, Murdoch University, Australia.

[10] Wilson, M. (1999). "Using words about thinking: Content analysis of chemistry teacher's Classroom talk". International Journal of Science Education, 21(10), 1067-1085, https://doi.org/10.1080/095006999290192 


$$
\text { المجلة الدولية للدراسات التربوية والنفسية }
$$

International Journal of Educational \& Psychological Studies (EPS)

Journal Homepage: https://www.refaad.com/views/EPSR/Home.aspx

www.refaad.com

ISSN: 2520-4149 (Online) 2520-4130 (Print)

\title{
Science teacher's perceptions and practicing the dimensions of scientific literacy at Ajloun educational directorate
}

\author{
1 Walaa Mustafa Al-Smadi, ${ }^{2}$ Abdullah Mohammad Khataibeh, ${ }^{3}$ Imad Tawfiq Al-Sadi \\ ${ }^{1} \mathrm{PhD}$ student, Faculty of Education, Department of Curricula and Teaching Methods of Science, Yarmouk University, \\ Jordan \\ 2,3 Professor, Faculty of Education, Department of Curricula and Teaching Methods of Science, Yarmouk University, \\ Jordan \\ ${ }^{1}$ Kulaep_hn@yahoo.com , 2 khataibeh@yu.edu.jo, 3 Imad.Sadi@yu.edu.jo
}

\begin{abstract}
:
The study aimed to identify the level of science teachers' understanding of the dimensions of scientific culture and their practice in light of some variables in the Ajloun Education Directorate. The study sample, it reached (200) male and female teachers, chosen by the stratified random method, and the study used the survey descriptive method, where the researcher used tools to collect Albia Nate through (a test of science teachers' understanding of the dimensions of scientific culture, and their practice of identifying them), and the results showed that the relative importance ranged between $(75 \%-51 \%)$, where science came first in the way of thinking with the highest relative importance (75\%) While the interaction of science, technology and society came in the last rank with relative importance amounted to (51\%), and the relative importance of the test of scientific culture as a whole (60\%), as it showed a high degree of the dimensions of scientific culture, also showed a positive relationship statistically between the understanding of science teachers of the dimensions Scientific culture and their practice there, and showed no differences in the level of teacher understanding Science for the dimensions of scientific culture according to specialization, years of experience, degree, lack of differences in their practice of dimensions of scientific culture according to specialization, years of service, and the presence of differences according to the variable scientific degree in favor of the degree Bachelor's Diploma. The study recommended training science teachers during their service and increasing interest in topics that develop the understanding of science such as the philosophy of science.
\end{abstract}

Keywords: Understanding; Science Teachers; Dimensions of Scientific Culture.

\section{References:}

[1] 'Bydat, Dwqan. (1998). Albhth Al'lmy Mfhwmh Wadwath Wasalybh. T6, 'Man: Dar Alfkr

[2] 'Lywh, Naryman Whsn, 'Bd Alrhmn. (2017). "Mstwa Fhm M'lmy Al'lwm Lmstwyat Althqafh Al'lmyh W'laqh Dlk Batjahathm Nhw Al'lwm". Atrwht Dktwrah Ghyr Mnshwrh. Jam't Alyrmwk. Erbd. Alardn.

[3] AAAS. (1993). "Benchmarks for Science Literacy: Project 2061". New York: Oxford University press.

[4] Aikenhead, S. (2006). "Science education for everyday- life: evidence- based practice". New York: Teachers College Press.

[5] American Association for the Advancement of Science (AAAS). (1990). Science for all Americans. New York: Oxford University press.

[6] Association for the Advancement of Science (AAAS). (1993). "Benchmarks for Science Literacy: A project 2061 Report". New York: Oxford University press.

[7] Bajbyr, 'Bdalqadr. (2003). "Mstwa Althqafh Al'elmyh Altknwlwjyh Walbd'eyh Lda Jam't Hdrmwt Ll'lwm Waltknwlwjya Wmsadr Aktsabhma". Rsalt Majstyr Ghyr Mnshwrh. Jam't Alyrmwk. Alardn.

[8] Alblwshy, Slyman Walmqbaly, Fatmh. (2006). "Athr Altdryb 'La Tsmym Jdwl Alastqsa' Fy Tdrys Al'lwm 'La 'Mlyat Al'lm Walthsyl Lda Tlamyd Alsf Altas' Mn Alt'lym Al'am Bsltnh 'Man". Mjlt Al'lwm Altrbwyh Walnfsyh: 7(1): 43-61.

[9] Chi, C. (2005). "First_YearPre_Service Teachers in Taiwan_ Do They enter the teacher program with Satisfactory scientific literacy and attitudes Toward?". International of Journal Science Education 27(13), 1549-1570, https://doi.org/10.1080/09585190500186401 
[10] Deng, Z. (2007). "Scientific Literacy as an Issue of Curriculum Inquiry". Promoting Scientific Literacy: Science Education Research in Transaction Proceedings of the Linnaeus Tercentenary Symposium held at Uppsala University, Uppsala, Sweden, 21-25 March 2007.

[11] Abw Dhb, Eyman. (2004). "Brnamj Ethra'ey Fy Al'lwm Almbsth Wathrh 'La Tnmyh B'd 'Nasr Althqafh Al'Imyh Walmharat Alajtma'yh Lda Tlamyd Alhlqh Alabtda'yh". Atrwht Dktwrah Ghyr Mnshwrh. Klyt Al'elwm Altrbwyh. Jam't Jnwb Alwady, Msr.

[12] Dlwl, Ayad. (2013). "Mstwa Fhm M'elmy Al'lwm Ltby'eh Al'elm Walastqsa' Al'lmy Wfq M'ayyr Nsta Bghzh". Rsalt Majstyr Ghyr Mnshwrh. Aljam'h Alaslamyh. Ghzh.

[13] Alhjry, Hsn. (2006). "Mstwa Fhm M'elmy Al'elwm Ltby't Al'lm W'laqth Bmmarsathm Alsfyh". Rsalt Majstyr Ghyr Mnshwrh. Jam't Alsltan Qabws. Sltnt Oman.

[14] Khtaybh, 'Bd Allh. (2008). T'lym Al'lwm Lljmy'. T2. 'Man: Dar Almsyrh Llnshr Waltwzy' Waltba'h.

[15] Lederman, G. (1992). "Students and teacher's conceptions of the nature of science: A re-assessment". School Science and Mathematics, 86, 91-99.

[16] Mclean, J. (1999). "Incorporating the use of Concept Maps and diagrams is student's formal lab report writing". Master thesis, Montma State University, USA.

[17] Murcia, K. (2006). "Scientific Literacy for Sustainability". PhD Thesis, Murdoch University, Australia.

[18] Alnmr, Mdht (1997). "Flsft Wmttlbat A'dad M'lm Al'elwm Llqrn Alhady Wal'shrwn". Alm'tmr Al'lmy Alawl, Almjld Alawl, Aleskndryh

[19] Nshwan, Y'qwb Hsyn. (2001). Aljdyd Fy T'lym Al'lwm. 'Man: Dar Al'rqan.

[20] Alqdah, Mhmd. (2016). "Mstwa Fhm Tby't Al'lm Wfq M'ayyr (Nsta) Lda M'Imy Al'lwm Fy Alardn Fy Dw' B'd Almtghyrat". Rsalt Majstyr Ghyr Mnshwrh. Jam't Al Albyt. Almfrq, Alardn.

[21] Alrhyly Wslwm Amynh (2017). "Mas Alkhal Al'lmy Fy Madh Alfyzya'". Ebda'at Trbwyh: 1(1): 127-133.

[22] Rwaqh, Ghazy Waltmymy, Rna. (2017). "Tby't Al'lm Walastqsa' Al'lmy Lda M'lmy 'Lwm Almrhlh Alasasyh Al'lya W'laqthma Bmstwa Alfhm Al'lmy Llqdaya Aljdlyh Walatjahat Al'lmyh". Atrwht Dktwrah Ghyr Mnshwrh. Klyt Altrbyh. Jam't Alyrmwk. Erbd, Alardn.

[23] Alrwys, 'Bd Al'zyz (2008). "Alnzryh Albna'yh Wt'lym Alryadyat. Tswr Mqtrh Mqdm Ala Alndwh Al'Imyh 'Lm Alnfs Wqdaya Altnmyh Alfrdyh Walmjtm'yh". Alryad. Klyt Altrbyh. Jam't Almlk S'wd.

[24] Alshhrany, 'Amr Wals'yd, S'yd. (1997). Tdrys Al'lwm Fy Al'lwm Al'am. Alryad: Jam't Almlk S'wd.

[25] Alshhrany, 'Amr. (2000). "Mstwa Althqafh Al'elmyh Lda Tlab Almstwyyn Alawl Walrab'e Mn Altkhssat Al'elmyh Bklyh Altrbyh Babha Wdwr Brnamj Ala'edad Fy Tnmyth". Mjlt Rsalh Alkhlyj: (75): 39-25.

[26] Alshmry, 'Bd Allh. (2014). "Dwr Mnahj Al'lwm Fy Tnmyh Almfahym Al'lmyh Wmharat Alastqsa' Al'lmy Lda Tlamyd Alsfwf Al'lya Balmrhlh Alabtda'yh". Rsalt Majstyr Ghyr Mnshwrh. Jam't Alqsym. Als'wdyh.

[27] Slah, Mndr. (2000). "Mstwa Aljanb Alm'erfy Llthqafh Al'lmyh Lda Tlbh Alsf Alawl Althanwy Fy Dw' Mtghyrat T'lymyh T'lmyh". Rsalt Majstyr Ghyr Mnshwrh. Jam't Alyrmwk. Alardn.

[28] Slym, Mhmd. (1998). "Adwa' 'La Ttwyr Mnahj Al'lwm Llt'lym Al'am Fy Aldwl Al'erbyh". Mjlt Altrbyh Al'Imyh: 1(2): 121145 .

[29] Alz'by, Tlal. (2008). "Mstwa Althqafh Al'lmyh Lda M'lmy Alfyzya' Fy Almrhlh Althanwyh W'laqth Bmstwa Althqafh Al'Imyh Walatjahat Nhw Al'Im Lda Tlbthm". Almjlh Alardnyh Ll'lwm Alttbyqyh: 11(1): 103 - 116.

[30] Alz'by, Tlal. (2010). "Athr Astkhdam Brnamj Astqsa'y Qa'm 'La Alnshat Alastqsa'y Fy Althsyl Almbashr Walm'jl Wtnmyh Mharat Altfkyr Al'lmy Walatjahat Al'lmyh Wfhm Tby't Al'lm Lda Talbat Tkhss M'lm Sf Fy Jam't Alhsyn Bn Tlal". Mjlt Aldrasat Altrbwyh Walnfsyh: 4(1): 16 - 41.

[31] Zytwn, 'Aysh. (2008). Asalyb Tdrys Al'lwm. 'Man: Dar Alshrwq Llnshr Waltwzy'.

[32] Zytwn, 'Aysh. (2010). Alatjahat Al'ealmyh Alm'easrh Fy Mnahj Al'lwm Wtdrysha. 'Man: Dar Alshrwq Llnshr Waltwzy'.

[33] Wilson, M. (1999). "Using words about thinking: Content analysis of chemistry teacher's Classroom talk". International Journal of Science Education, 21(10), 1067-1085, https://doi.org/10.1080/095006999290192. 